\title{
La vittima nel procedimento de libertate: i precari equilibri di un nuovo protagonismo ancora troppo poco meditato
}

\author{
The crime victim in bail proceedings: the uncertain \\ balance of a new but still unmeditated protagonism
}

\section{Lina Caraceni ${ }^{1}$}

Università degli Studi di Macerata, Italia

lina.caraceni@unimc.it

https://orcid.org/0000-0002-8433-5427

\begin{abstract}
Una nuova sensibilità per le istanze di cui è portatrice la vittima del reato ha determinato un rafforzamento del suo ruolo nell'ambito del procedimento penale in una duplice dimensione: come destinataria di misure atte a proteggerla nel processo (vittimizzazione reiterata) e dal processo (vittimizzazione secondaria) e come soggetto attivo, capace di esercitare diritti e facoltà a tutela della propria integrità psico-fisica. E il sistema di protezione è stato costruito guardando prioritariamente alle vittime vulnerabili, nel perimetro delle indicazioni fornite dal diritto dell'Unione europea. I principali interventi di riforma hanno interessato la materia cautelare: sono state introdotte misure pro victima (gli artt. 282bis e 282-ter c.p.p.) ed è stato riconosciuto alla persona offesa un diritto all'informazione sull'evoluzione dello status custodiae dell'accusato (artt. 90-ter, 282-quater e 299 comma 2-bis c.p.p.) a cui è associato un diritto di interlocuzione nel procedimento per la revoca e la sostituzione di una misura cautelare (artt. 299 commi 3 e 4-bis c.p.p.). L'analisi sarà condotta su questo secondo aspetto,
\end{abstract}

1 Professore associato di diritto processuale penale nell'Università di Macerata, dove insegna Diritto processuale penale e Diritto penitenziario. Ha conseguito un PhD in Diritto processuale penale ed indirizzato i suoi studi sui temi del carcere, della tutela dei soggetti deboli e del diritto probatorio. Autrice di saggi, articoli e monografie, ha pubblicato Poteri d'ufficio in materia probatoria e imparzialità del giudice penale (Giuffrè, 2007) e curato L'esecuzione delle pene nei confronti dei minorenni. Commento al d.lgs. 2 ottobre 2018, n. 121 (con M.G. Coppetta, Giappichelli, 2019). È stata componente del tavolo XII degli Stati generali dell'esecuzione penale e della Commissione ministeriale per la riforma dell'ordinamento penitenziario minorile. 
evidenziando che si è trattato di scelte normative che assegnano alla vittima un nuovo protagonismo sulla scena processuale. Peccato che la traduzione normativa di tali intendimenti non abbia sortito gli effetti sperati: un lessico giuridico non sempre impeccabile, unito ad una scarsa sistematicità di novelle ripetute, non soltanto mettono in discussione l'obiettivo di protezione della vittima, ma rischiano anche di neutralizzare quel sistema di garanzie per l'accusato su cui si regge il procedimento cautelare.

Parole ChIaVe: Vittima; Protezione; Procedimento cautelare; Diritto all'informazione; Diritto di intervento; Procedimento di revoca.

ABStRACT: A renewed attention on the crime victim's issues has led to strengthen her/his role in criminal proceedings in two dimensions: as holder of the right to be protected in the process (repeated victimization) and from the process (secondary victimization) and as an active subject, capable of exercising rights and faculties to protect her/his psycho-physical integrity. The protection system was built on vulnerable victims, taking into account the indications of European Union law. The main reform has seen the introduction of pro victima measures in the pre-trial stage (artt. 282-bis and 282-ter c.p.p.) and the victim right to information on the evolution of the custody status of the accused (artt. 90-ter, 282-quater and 299 paragraph 2-bis c.p.p.) which is associated with a right to participate in the procedures for the enforcement and disposal of the measures (artt. 299 paragraph 3 and 4-bis c.p.p.). The analysis will focus on this second aspect, highlighting that the procedural law gives a new key role to the victim. Unfortunately, the hoped-for results have not been achieved: unclear legal language and lack of coordination between the various reforms have compromised the goal of protecting the victim and have introduced the risk that guarantees provided to the accused in the decision-making procedures about her/his personal liberty will be neutralized.

KeYwords: Crime victim; Protection; Bail proceedings; Right to information; Right to participate; Personal liberty of the accused person.

Sommario: 1. Breve premessa definitoria e quadro normativo di riferimento. - 2. Il diritto all'informazione sull'evoluzione dello status custodiae: significati e ambito di applicazione. - 3. La duplice dimensione della comunicazione "rafforzata" alla persona offesa. 4. Natura e ampiezza dell'intervento della vittima nel procedimento di revoca delle misure. -5 . I riflessi della disciplina sui diritti di libertà del soggetto in vinculis. - 6. Conclusioni: prospettive di riforma. 


\section{BREVE PREMESSA DEFINITORIA E QUADRO NORMATIVO DI RIFERIMENTO.}

In anni recenti, una marcata sensibilità per gli interessi di cui è portatrice la vittima del reato ha contribuito a rimodellare il processo penale, in particolare il ruolo assegnato ai suoi protagonisti e ha consentito all'"attore privato" di uscire dal cono d'ombra dell'egemone figura dell'accusatore pubblico in cui per secoli è stato relegato ${ }^{2}$, mostrandosi con il volto fragile, sofferente di una persona "in carne ed ossa" che rivendica attenzione e chiede garanzie per i propri diritti ${ }^{3}$. Si tratta di approdi normativi attinti sotto la spinta di una mutata temperie culturale, favorita da una vivace legislazione europea ${ }^{4}$ che si è assunta l'arduo compito di governare il processo di rafforzamento di una comune politica criminale tesa a valorizzare le istanze (anche private) di chi subisce le conseguenze del reato ${ }^{5}$.

2 Cfr. LUPARIA, Luca, Quale posizione per la vittima nel modello processuale italiano?, in AA.VV., Lo scudo e la spada. Esigenze di protezione e poteri delle vittime nel processo penale tra Europa e Italia, Torino, 2012, p. 20 ss.; VENTUROLI, Marco, La tutela della vittima nelle fonti europee, in Dir. pen. cont., 2012, f. 3-4, p. 86 ss.

3 GUERRA, Mariaemanuela, Questioni in tema di diritti di informazione ed interlocuzione della persona offesa nell'incidente cautelare, in Cass. pen., 2017, p. 2537.

4 La più recente prospettiva vittimologica rintracciabile nella normativa continentale, che molto ha influito su quella nazionale, inizia con la decisione quadro 2001/220/GAI (assunta dal Consiglio d'Europa il 15 marzo 2001) e si compie con la direttiva 2012/29/UE (adottata dal Parlamento e dal Consiglio d'Europa il 25 ottobre 2012), alla quale va assegnato il merito di aver composto un organico corredo di diritti e garanzie, cui gli ordinamenti nazionali debbono dare attuazione, per consentire alla vittima di svolgere un ruolo effettivo e adeguato nelle dinamiche dell'accertamento penale e ricevere un trattamento in grado di salvaguardarne la dignità. La direttiva 2012/29/UE è stata recepita in Italia con il d.lgs. 15 dicembre 2015, n. 212. In argomento, ALLEGREZZA, Silvia, Il ruolo della vittima nella direttiva 2012/29/UE, in AA.VV., Lo statuto europeo delle vittime di reato. Modelli di tutela tra diritto dell'Unione e buone pratiche nazionali, a cura di LUPARIA, Luca, Padova, 2015, p. 3 ss.

5 Per la direttiva 2012/29/UE è vittima sia «la persona fisica che ha subito un danno, anche fisico, mentale o emotivo, o perdite economiche che sono stati causati direttamente da un reato», sia il «familiare di una persona la cui 
Peraltro, questo percorso non è stato così lineare come potrebbe apparire; anzi in maniera rapsodica a guadagnare l'attenzione legislativa sono stati particolari soggetti che, per le loro caratteristiche personali o per la cifra criminale dei reati commessi nei loro confronti, chiedevano di occupare sulla scena processuale uno spazio più consono a soddisfare i loro bisogni ${ }^{6}$. Lo statuto europeo della vittima (che ritroviamo nella direttiva 2012/29/UE) è stato costruito partendo da chi, nella politica penale continentale di questi anni, ha rappresentato la priorità delle priorità: «le vittime del terrorismo, della criminalità organizzata, della tratta di esseri umani, della violenza di genere, della violenza nelle relazioni strette, della violenza o dello sfruttamento sessuale o dei reati basati sull'odio e le vittime con disabilità ${ }^{7}$. In particolare, è la lotta a reati particolarmente odiosi come quelli che attentano all'integrità psicofisica e alla libertà sessuale di donne e minori ad aver catturato l'attenzione del nomoteta europeo,

morte è stata causata direttamente da un reato e che ha subìto un danno in conseguenza della morte di tale persona».

6 Si tratta delle vittime vulnerabili, anche se la categoria è di difficile inquadramento, stante la mancanza di una nozione normativa condivisa: BUZZELLI, Silvia, La fragilità probatoria del dichiarante minorenne e la giustizia penale in Europa, in CESARI, Claudia (a cura di), Il minorenne fonte di prova nel processo penale, Milano, 2015, p. 1 ss.

Nutrito l'elenco delle iniziative legislative europee volte a rivalutare la figura dell'offeso e il suo ruolo processuale partendo dai vulnerabili: la Convenzione di Lanzarote sulla protezione dei minori dallo sfruttamento e dagli abusi sessuali, siglata il 25 ottobre 2007 (l'Italia ha provveduto al recepimento con la $1.1^{\circ}$ ottobre 2012, n. 172) e la Convenzione di Istanbul sulla prevenzione e la lotta alla violenza contro le donne e la violenza domestica, firmata l'11 maggio 2011 (l'Italia ha provveduto alla ratifica con la l. 27 giugno 2013, n. 77). E non vanno dimenticate le direttive del Consiglio d'Europa: la 2011/36/UE del 5 aprile 2011, relativa alla prevenzione e la repressione della tratta di esseri umani e la protezione delle vittime, la 2011/92/UE del 13 dicembre 2011, riguardante la lotta contro l'abuso e lo sfruttamento sessuale dei minori e la pornografia minorile e la 2011/99/UE del 13 dicembre 2011 sull'ordine di protezione europeo. Per una ricostruzione della legislazione europea sul punto, VENTUROLI, Marco, La tutela della vittima nelle fonti europee, in Dir. pen. cont., 2012, f. 3-4, p. 86 ss., nonché SECHI, Paola, Vittime di reato e processo penale: il contesto sovranazionale, in Cass. pen., 2017, p. 850 ss. e 1236 ss.

7 Art. 22 comma 3 direttiva 2012/29/UE. Sul punto, GIALUZ, Mitja, Lo statuto europeo delle vittime vulnerabili, in AA.VV., Lo scudo e la spada. Esigenze di protezione e poteri delle vittime nel processo penale tra Europa e Italia, Torino, 2012 , p. 60 
una sfida combattuta a suon di ripetuti e, a volte, sovrapposti interventi che hanno guardato ora ai diritti individuali, ora ai bisogni di protezione, ora alle istanze di partecipazione al processo penale, smarrendo non di rado quello sguardo d'insieme, quella sistematicità che non può mancare quando si ha l'ambizione di "armonizzare" sistemi giuridici differenti, di dettare prescrizioni comuni e vincolanti per gli ordinamenti statuali ${ }^{8}$.

E le ricadute sul fronte interno non si sono fatte attendere: la nostrana legislazione penale, chiamata ad attuare quelle indicazioni, ha dato vita a interventi altrettanto rapsodici - e non sempre in perfetta consonanza -, con conseguenti problemi di organicità e applicazione generalizzata ${ }^{9}$ che non si può dire abbiano contribuito efficacemente a rafforzare la posizione della vittima in quanto tale, non identificata cioè da una "particolare etichetta". E già nella trama normativa del codice di procedura penale del 1988 la vittima non brillava per protagonismo, malgrado vi fosse stata, quantomeno dal punto di vista lessicale, una chiara scelta di campo nella definizione del suo status. Si era optato per l'uso della locuzione «persona offesa», perché sembrava meglio identificare la dimensione giuridico-soggettiva di cui gode chi subisce l'aggressione al bene protetto: non soltanto una persona bisognevole di tutela (una vittima appunto), ma un soggetto titolare di diritti e aspettative al quale l'ordinamento riconosce un preciso ruolo nell'accertamento della condotta lesiva. Peccato che l'espressione sia rimasta vanamente declamatoria, non corrispondendo ad essa uno strumentario processuale in grado di assicurare una partecipazione tale da rendere effettiva la salvaguardia della propria posizione giuridica ${ }^{10}$.

8 ALLEGREZZA, Silvia, La riscoperta della vittima nella giustizia penale europea, in AA.VV., Lo scudo e la spada. Esigenze di protezione e poteri delle vittime nel processo penale tra Europa e Italia, Torino, 2012, p. 5.

9 IASEVOLI, Clelia, Pluralismo delle fonti e modifiche al c.p.p. per i delitti commessi con violenza alla persona, in Dir. pen. e proc., 2013, p. 1392.

10 E a proposito della confusione che regna sul ruolo dell'offeso nel rito penale, si segnala l'espressione impiegata nell'art. 282-quater c.p.p. (e già apparsa nell'art. 472 comma 3-bis c.p.p.): nell'assolvimento del dovere di comunicazione dell'evolvere dello status cautelare dell'accusato il destinatario viene identificato come "parte offesa», quando la vittima, in senso tecnico, parte può esserlo soltanto con la costituzione di parte civile e dunque, vantando un diritto alla restituzione o al risarcimento del danno. Un infortunio semantico 
Certo, rispetto al passato crescono i diritti processuali della vittima, perché si ampliano gli interessi di cui è portatrice: non soltanto (come da tradizione) contribuisce all'accertamento del reato e alla punizione del reo, ma ambisce a difendere per il tramite del processo penale anche la propria persona ${ }^{11}$. Tre le direttrici che ne hanno disegnato il nuovo ruolo: partecipazione ai percorsi di diversion ${ }^{12}$ e/o "ristorativi"13; informazione e misure di sostegno in snodi cruciali del procedimento penale $^{14}$; protezione nel processo da possibili fenomeni di vittimizzazione primaria (pericoli che possono di nuovo provenire dal presunto autore del reato) e protezione dal processo e da ogni forma di vittimizzazione secondaria (ripercussioni negative nel momento in cui l'offeso è chiamato a fornire il suo contributo all'accertamento penale) ${ }^{15}$.

Nei più recenti interventi normativi, quelli più marcatamente rivolti alle vittime vulnerabili, il processo penale è diventato il terreno elettivo di protezione dell'offeso, orientando così la giurisdizione penale «verso funzioni di controllo sociale, o meglio, di garanzia di solidarietà

che nulla aggiunge ai diritti e alle facoltà che come persona offesa può esercitare. Cfr. DI NUZZO, Viviana, Giudizio cautelare e tutela della persona offesa, tra istanze di protezione e prospettive di partecipazione attiva, in Legislaz. pen., 29.7.2020, p. 5.

11 RECCHIONE, Sandra, La vittima cambia il volto del processo penale: le tre parti "eventuali", la testimonianza dell'offeso vulnerabile, la mutazione del principio di oralità, in Dir. pen. cont., 16 gennaio 2017, p. 1.

12 Per un approfondimento, v. ORLANDI, Renzo, I diritti della vittima in alcune particolari modalità di definizione del rito penale, in BARGIS, Marta - BELLUTA, Hervè (a cura di), Vittime di reato e sistema penale. La ricerca di nuovi equilibri, Torino, 2017, p. 167 ss.

13 PATANÈ, Vania, Percorsi di giustizia riparativa nel sistema penale italiano, in BARGIS, Marta - BELLUTA, Hervè (a cura di), Vittime di reato e sistema penale. La ricerca di nuovi equilibri, Torino, 2017, p. 545 ss.

14 Privilegiato il terreno della fase investigativa e della vicenda de libertate: v., anche per gli accenti critici, PAULESU, Pier Paolo, Vittima del reato e processo penale: uno sguardo d'insieme (informazioni, diritti, tutele), in BARGIS, Marta - BELLUTA, Hervè (a cura di), Vittime di reato e sistema penale. La ricerca di nuovi equilibri, Torino, 2017, p. 134 ss.

15 Sulla classificazione delle forme di vittimizzazione, VENTUROLI, Marco, La vittima nel sistema penale: dall'oblio al protagonismo?, Napoli, 2015, pp. 51 ss. 
sociale, che istituzionalmente non le appartengono» ${ }^{16}$. Gli strumenti dell'accertamento penale, che sulla carta vengono forgiati, aggiustati e sagomati per perseguire il nobile intento di proteggere i più deboli da deplorevoli condotte violente, sottotraccia sono piegati alla soddisfazione di istanze (nemmeno tanto nascoste) di difesa sociale. Indicativo, in tal senso, il preambolo del d.1. 14 agosto 2013, n. 93, la novella di interesse ai nostri fini, quella che più di altre ha riscritto la disciplina del procedimento cautelare nel segno delle vittime di violenza domestica e di genere ${ }^{17}$ : per il legislatore «il susseguirsi di eventi di gravissima efferatezza in danno di donne e il conseguente allarme sociale che ne è derivato rendono necessari interventi urgenti miranti ad inasprire, per finalità dissuasive, il trattamento punitivo degli autori di tali fatti, introducendo, in determinati casi, misure di prevenzione finalizzate alla anticipata tutela delle donne e di ogni vittima di violenza domestica [...]». Le espressioni «allarme sociale», «finalità dissuasive» e «misure di prevenzione» svelano le reali ragioni della novella.

Evidente il rischio che si corre: che la vittima venga processualmente "piegata" al conseguimento di obiettivi politici che poco hanno a che fare con la sua promozione quale soggetto portatore di interessi, aspettative di natura giudiziale. Questo approccio è oltremodo manifesto proprio in materia de libertate, dove l'apparato della coercizione ante iudicatum viene sovente strumentalmente utilizzato dal legislatore dell'emergenza per sedare l'impatto emotivo del reato sulla vittima e di riflesso (qui si rivela l'interesse politico) sulla collettività, perché consente di rispondere fin da subito all'ansia di giustizia dei consociati che chiedono risultati rapidi ed esemplari, poco importa se ottenuti ricorrendo ad anticipati surrogati punitivi - le misure cautelari appunto, magari nella forma più emblematica e rassicurante del carcere ${ }^{18}$ - distolti perciò dalla loro naturale

16 PAULESU, Pier Paolo, Vittima del reato e processo penale: uno sguardo d'insieme (informazioni, diritti, tutele), in BARGIS, Marta - BELLUTA, Hervè (a cura di), Vittime di reato e sistema penale. La ricerca di nuovi equilibri, Torino, 2017, p. 133.

17 Il d.1. 93/2013, recante disposizioni urgenti in materia di sicurezza e per il contrasto della violenza di genere, è stato conv. nella l. 16 agosto 2013, n. 119.

18 LORUSSO, Sergio, Le conseguenze del reato. Verso un protagonismo della vittima nel processo penale?, in Dir. pen. proc., 2013, p. 883. 
funzione di rimedi contro i pericula che possono mettere in discussione l'accertamento penale, nel rispetto della presunzione di non colpevolezza e della inviolabilità della libertà personale dell'accusato ${ }^{19}$.

Ci si muove su un crinale davvero impervio, poiché un sistema che si sofferma a guardare con troppa insistenza agli interessi giuridici della vittima rischia di perdere di vista quelli dell'imputato: eventuali derive "vittimocentriche", in epoca di populismo penale, non solo non darebbero soddisfazione alle legittime attese delle persone offese, ma vieppiù «potrebbero risultare, per l'imputato, irragionevolmente inique e, comunque, costituzionalmente eccepibili $»^{20}$.

Non si può negare che alcuni degli interventi normativi operati sul terreno delle misure cautelari rappresentino un deciso passo avanti per il rafforzamento della posizione della vittima, soprattutto sul versante dei suoi bisogni di protezione ${ }^{21}$; protezione declinata in un duplice significato: l'uno, in una dimensione criminologica, guarda all'offeso come soggetto passivo, destinatario di interventi miranti a salvaguardarne l'integrità psico-fisica (si veda l'introduzione delle misure cautelari di cui agli artt. 282-bis e 282-ter c.p.p.) ${ }^{22}$; l'altro, riconducibile ad una logica più

19 MORELLI, Francesco, Commento all'art. 9 d.1. 23 febbraio 2009, n. 11, in Legislaz. pen., 2009, p. 509.

20 PAULESU, Pier Paolo, Vittima del reato e processo penale: uno sguardo d'insieme (informazioni, diritti, tutele), in BARGIS, Marta - BELLUTA, Hervè (a cura di), Vittime di reato e sistema penale. La ricerca di nuovi equilibri, Torino, 2017 , p. 131. V. anche RAFARACI, Tommaso, Tutela della vittima nel sistema penale delle garanzie, in Criminalia, 2010, p. 273, secondo cui la troppa enfasi posta sulle esigenze di tutela della posizione della vittima potrebbe «convertire il processo nel luogo in cui comminare di fatto atipiche sanzioni anticipate, e su cui scaricare istanze di prevenzione e di "sicurezza"».

Brevemente, ecco i provvedimenti più significativi: oltre al d.1. 93/2013 e al d.lgs. 212/2015 già richiamati, la 1.4 aprile 2001, n. 154 , riservata alla lotta contro la violenza nelle relazioni familiari, il d.1. 23 febbraio 2009, n. 11, conv. con modif. nella 1. 23 aprile 2009, n. 38, recante misure urgenti in materia di sicurezza pubblica e di contrasto alla violenza sessuale, nonché in tema di atti persecutori, il d.lgs. 11 febbraio 2015, n. 9, sull'ordine di protezione europeo e la 1. 19 luglio 2019, n. 69 recante modifiche al codice penale, al codice di procedura penale e altre disposizioni in materia di tutela delle vittime di violenza domestica e di genere (c.d. Codice rosso).

22 In argomento, tra i molti, BONINI, Valentina, Il sistema di protezione della vittima e i suoi riflessi sulla libertà personale, Padova, 2018, p. 169 ss., nonché, 
strettamente processuale, lo vuole protagonista, soggetto attivo che partecipa alla vicenda giudiziaria attraverso l'esercizio di poteri e facoltà in grado di influire sulle sorti del rito ( $s$ fa riferimento sia ai doveri informativi nei confronti dell'offeso ex artt. 90-ter e 282-quater c.p.p., che alla composita disciplina dell'art. 299 c.p.p. riguardante l'intervento della vittima nel procedimento di revoca o sostituzione di una misura cautelare). E su questo protagonismo verrà focalizzata la nostra attenzione, con un'avvertenza metodologica: ci si propone non soltanto una verifica del raggiungimento degli specifici obiettivi perseguiti con le disposizioni introdotte, ma queste verranno sottoposte ad una "prova di resistenza" per testare la tenuta della nuova dimensione partecipativa dell'offeso in contrapposizione all'accusato nel peculiare contesto cautelare, dove massima è la salvaguardia dei diritti di libertà di chi subisce la pretesa punitiva statuale.

\section{IL DIRITTO ALL'INFORMAZIONE SULL'EVOLUZIONE DELLO STATUS CUSTODIAE: SIGNIFICATI E AMBITO DI APPLICAZIONE.}

La disciplina processuale di protezione della vittima trova nel diritto all'informazione una premessa imprescindibile: nella vicenda cautelare ha ad oggetto la conoscenza dell'evolvere dello status libertatis dell'accusato e consente all'offeso, da un lato, di adottare le necessarie misure per difendersi dagli eventuali pericoli che potrebbero derivare da un "allentamento" del vincolo coercitivo; dall'altro, di intervenire consapevolmente ogni qual volta si discuta di questo "allentamento". Come riconosce la direttiva 2012/29/UE, che dà precise indicazioni in merito, deve essere assicurata alla vittima «la possibilità di essere informata, senza indebito ritardo, della scarcerazione o dell'evasione della persona posta in stato di custodia cautelare, processata o condannata», nonché delle «eventuali pertinenti misure attivate per la sua protezione», con l'obiettivo di salvaguardarla «da vittimizzazione

volendo, CARACENI, Lina, Misure cautelari pro victima e diritti di libertà dell'accusato: a proposito di una convivenza faticosa, in Arch. n. proc. pen., 2017, p. 254 ss. 
secondaria e ripetuta, intimidazioni e ritorsioni, compreso il rischio di danni emotivi e psicologici ${ }^{23}$.

Plurime le disposizioni codicistiche a ciò dedicate: una previsione di più ampia portata nel contesto de libertate, quella dell'art. 90-ter c.p.p. e altre che innalzano il livello di tutela in snodi particolari della procedura (gli artt. 282-quater e 299 commi 2-bis, 3 e 4-bis c.p.p.). Mentre la prima è quasi una norma-bandiera che sancisce il diritto della vittima, dietro sua manifestazione di interesse, di conoscere il momento in cui il prevenuto, per ragioni diverse, dovesse tornare in libertà, le altre sembrano profilare, almeno sulla carta, un'informazione che assegna all'offeso maggiore "visibilità processuale" nell'incidente cautelare $^{24}$. Tuttavia, testi non certo impeccabili rischiano di rendere meramente declamatorie queste disposizioni; addirittura, su alcuni aspetti, il diritto all'informazione presenta un'ipertrofia nemica dell'efficacia, probabilmente dovuta alla sovrapposizione di interventi di modifica non sempre adeguatamente coordinati.

Partiamo dall'art. 90-ter c.p.p. e dalla delimitazione del perimetro entro cui sono salvaguardate le prerogative informative riconosciute alla vittima. La norma prevede la possibilità per l'offeso, il quale tema per la propria vita e incolumità, di essere informato per il tramite della polizia giudiziaria dei «provvedimenti di scarcerazione e di cessazione della misura di sicurezza detentiva» e «dell'evasione dell'imputato in stato di custodia cautelare o del condannato nonché della volontaria sottrazione dell'internato all'esecuzione della misura di sicurezza detentiva». Il tutto nei casi in cui si proceda per «delitti commessi con violenza alla persona»e salvo che risulti «il pericolo concreto di un danno per l'autore del reato» ${ }^{25}$.

23 Cfr. l'art. 6, commi 5 e 6 e l'art. 18. Analoghe indicazioni vengono dalla Convenzione di Istanbul che nel mirare a far sì che gli Stati forniscano adeguata protezione alle vittime di violenza di genere, stabilisce il diritto di essere informate «dell'eventuale evasione dell'autore del reato, nonché della liberazione di quest'ultimo in via temporanea o definitiva»: art. 56 lett. $b$.

24 Così, anche BONINI, Valentina, Il sistema di protezione della vittima e $i$ suoi riflessi sulla libertà personale, Padova, 2018, p. 353.

25 Questa formula di salvaguardia finale, che estende i suoi effetti anche al procedimento di revoca e viene mutuata dall'art. $6 \S 6$ direttiva 2012/29/UE, impone di operare un bilanciamento tra le legittime attese della vittima e quelle del prevenuto: esclude l'assolvimento del dovere di comunicazione nei 
I doveri informativi, dunque, sono attivati da una richiesta dell'offeso ${ }^{26}$, giustificata dal fatto che mentre lo status detentivo dell'autore del reato gli consente di sentirsi relativamente "tranquillo", «deve essere prontamente messo "all'erta”, laddove tale condizione di carcerazione venga meno» ${ }^{27}$. Peraltro, se si è in presenza di uno dei reati di cui al comma 1-bis dello stesso articolo $^{28}$, la comunicazione è sempre dovuta e va effettuata tanto alla vittima che al suo difensore, se nominato. L'onere comunicativo grava sull'autorità giudiziaria che procede e viene assolto per il tramite della polizia giudiziaria.

Più di un dubbio esegetico rivela la formulazione della norma, a cominciare dall'uso del termine «scarcerazione». In senso tecnico, questo designa la cessazione di un provvedimento limitativo della libertà personale che consente al ristretto di riguadagnare la libertà (arg. ex art. 307 c.p.p.) e letto unitamente all'incipit della disposizione «fermo quanto previsto dall'articolo 299», dovrebbe riferirsi alle sole vicende riguardanti l'esecuzione della pena, poiché quelle che modificano il titolo cautelare sono affidate a quest'ultima disposizione, anche se la disciplina della sostituzione o della revoca non esaurisce tutti i casi in cui si assiste al venir meno della coercizione. In effetti, non vi sarebbe un dovere di informazione in favore della vittima, ad esempio, per le ipotesi di estinzione automatica determinata dallo spirare dei diversi termini, lacuna che parte della dottrina ritiene sia stata colmata proprio dall'introduzione dell'art. 90-ter c.p.p. ${ }^{29}$.

casi in cui il pericolo di danno riguardi l'accusato che, una volta liberato, rischi concretamente di subire azioni vendicative da parte della stessa vittima, animata dall'intento di farsi giustizia da sé.

26 Scelta opportuna, che tiene conto della volontà della vittima di poter essere ignorata nel procedimento penale (c.d. diritto all'oblio), come richiesto dall'art. 6, § 4 direttiva 2012/29/UE.

27 ZARCONE, Chiara, La vittima: quale spazio nel processo penale, in Quest. giust., 7 ottobre 2016, p. 14.

28 La disposizione è stata aggiunta dalla legge 69/2019 e riguarda le fattispecie previste dagli artt. 572, 609-bis, 609-ter, 609-quater, 609-quinquies, 609-octies e 612-bis c.p., nonché dagli artt. 582 e 583-quinquies c.p. nelle ipotesi aggravate ai sensi degli artt. 576 comma 1, nn. 2, 5 e 5.1, e 577 comma 1, n.1 e comma 2 c.p. (si tratta dei cc.dd. delitti da codice rosso).

29 ZACCHÈ, Francesco, Il sistema cautelare a protezione della vittima, in BARGIS, Marta - BELLUTA, Hervè (a cura di), Vittime di reato e sistema penale. 
Concludendo sull'apparato informativo in executivis e restando fedeli al significato più rigoroso del termine «scarcerazione», si dovrebbe arrivare ad escludere che la comunicazione sia dovuta in caso di benefici penitenziari (permessi di vario tipo, licenze) o di misure alternative alla detenzione che non sono provvedimenti idonei a far cessare lo stato detentivo ${ }^{30}$, senza tacere che una lettura così severa tradirebbe la ratio sottesa agli obblighi informativi: mettere in condizione la vittima di tutelarsi dalla reiterazione di future condotte violente da parte del presunto aggressore che ha recuperato, a qualunque titolo, maggiori spazi di libertà. E consapevole di ciò il legislatore sembra aver abbracciato un'esegesi estensiva del lemma «scarcerazione» con l'inserimento nell'art. 659 c.p.p. del comma 1-bis, a mente del quale quando il giudice di sorveglianza adotta un qualche provvedimento che determina la scarcerazione del condannato per uno dei reati da "codice rosso" (maltrattamenti in famiglia, atti persecutori, delitti contro la libertà sessuale), il pubblico ministero, per il tramite della polizia giudiziaria, deve darne immediata comunicazione alla persona offesa e, ove nominato, al suo difensore ${ }^{31}$.

Ancora più problematica appare l'esplicitazione del concetto di «violenza alla persona», che connota i delitti che darebbero diritto all'informativa; una categoria che non ha definizione normativa e che rischia di creare applicazioni arbitrarie a seconda dell'esegesi più o meno ampia a cui ricorrerà l'interprete. Oltretutto, l'ampiezza del significato dipende pure dal contesto in cui la delimitazione opera ${ }^{32}$. Per ciò che qui interessa, lo anticipiamo subito, una interpretazione restrittiva, nell'ottica della vittima, sterilizzerebbe i propositi di tutela connessi alla comunicazione. Oltretutto, questa peculiare categoria di reati non è rintracciabile nella normativa europea come fonte «per azionare uno

La ricerca di nuovi equilibri, Torino, 2017, p. 432-433; per ulteriori approfondimenti, v. infra., § 3 .

30 Così, SPAGNOLO, Paola, Nuovi diritti informativi per la vittima di reato, in Legislaz. pen., 4-7-2016, p. 18.

31 Il comma è stato introdotto dalla legge n. 69 del 2019.

32 Il riferimento a queste fattispecie lo ritroviamo nel procedimento di archiviazione (quando al pubblico ministero, ex art. 408 comma 3-bis c.p.p. è imposto di notificare comunque alla vittima la richiesta di archiviazione) e nel procedimento di revoca di una misura cautelare (art. 299 c.p.p., su cui infra, $\S \S 4$ e 5$)$. 
statuto di garanzie rafforzato» ${ }^{33}$. La direttiva 2012/29/UE non si affida alla natura del reato, ma «adotta un approccio più duttile e attento alle peculiarità delle singole fattispecie» ${ }^{34}$ prevedendo che il diritto all'informazione sullo status libertatis dell'accusato vada riconosciuto, previa richiesta dell'interessato, «almeno nei casi in cui sussista un pericolo o un rischio concreto di danno nei confronti della vittima» $»^{35}$ o comunque in presenza di «gravi reati ${ }^{36}$. E il «rischio concreto di danno», concetto non definibile in astratto, dovrebbe comprendere fattori quali la natura e la gravità del reato e il pericolo di ritorsioni. Pertanto, non dovrebbe operare nelle «situazioni in cui siano stati commessi reati minori e vi sia quindi soltanto un debole rischio di danno per le vittime» ${ }^{37}$.

Diversamente, il nostro legislatore, in sede di attuazione della direttiva, è ricorso ad un metodo classificatorio che "ingabbia" le fattispecie nei cui confronti operano queste peculiari garanzie, riaffermando, in questo modo, l'esistenza nella delicata materia cautelare, di una discrezionalità guidata dalla determinazione ex lege di condizioni, presupposti e criteri di valutazione oggettivi che favoriscano un intervento giurisdizionale quanto più possibile meditato, uniforme e tempestivo (i tempi della risposta giudiziale sono essenziali quando si pretende, come in questo caso, di garantire una qualche forma di protezione) $)^{38}$. L'operazione, pur condivisibile, non appare riuscita. In ambito penale, già la definizione dell'area semantica dell'espressione «violenza alla persona» divide da tempo la dottrina, la quale rileva come il codice penale, da un lato, definisca soltanto la violenza sulle cose e non sulle persone e, dall'altro, impieghi la nozione di violenza in varie accezioni,

33 GUERRA, Mariaemanuela, Questioni in tema di diritti di informazione ed interlocuzione della persona offesa nell'incidente cautelare, in Cass. pen., 2017, p. 2540.

34 CIAMPI, Stefano, Il diritto di difesa e all'informazione, in BARGIS, Marta BELLUTA, Hervè (a cura di), Vittime di reato e sistema penale. La ricerca di nuovi equilibri, Torino, 2017, p. 294.

35 Art. 6 § 6 direttiva 2012/29/UE e art. 56 Convenzione di Istambul.

36 Art. 11 direttiva 2012/29/UE.

37 Così la direttiva 2012/29/UE al punto 32 del considerato.

38 Su questi profili, v. BONINI, Valentina, Il sistema di protezione della vittima e $i$ suoi riflessi sulla libertà personale, Padova, 2018, p. 363. 
funzionali a soddisfare plurime esigenze di definizione normativa ${ }^{39}$. In questo contesto, la scelta della locuzione violenza ad personam sembra fungere «da elemento di compromesso, estendendo l'ambito dei doveri comunicativi oltre la sfera dei delitti germogliati dal rapporto personale tra vittima e imputato, ma, al contempo, restringendolo rispetto ad una esecrabile generalizzazione» ${ }^{40}$. Perché senza dubbio l'espressione va delimitata attraverso la ratio legis che ne ha determinato l'introduzione, vale a dire la tutela delle vittime nei reati connotati da violenza di genere e nelle relazioni familiari ${ }^{41}$.

Peccato che la perimetrazione dei significati da attribuire all'espressione resti assai sfuggente, anche affidandosi ai nobili fini che ne hanno ispirato l'adozione. Due sono le teorie che si contrappongono in merito: una adotta un concetto "spiritualizzato" e fortemente estensivo di violenza ${ }^{42}$, secondo cui sarebbe da considerare tale ogni atto che comporta l'impiego di una vis corpore corpori afflicta derivante da una qualsiasi azione (anche esercitata ricorrendo all'uso di mezzi fisici) idonea a coartare la volontà del soggetto passivo, compromettendone le capacità di determinazione ${ }^{43}$; l'altra, invece, si aggancia ad un concetto di violenza in senso stretto, da intendersi come aggressione fisica, come lesione o immediata esposizione a pericolo dei beni più direttamente attinenti alla dimensione fisica della persona, quali la vita, l'integrità o la

39 Sulla poliedricità del concetto, cfr. DE SIMONE, Giulio, voce Violenza, in Enc. dir., 1993, vol. XLVI, p. 903 s.; v. anche VIGANÒ, Francesco, La tutela penale della libertà individuale, vol. I, L'offesa mediante violenza, Milano, 2002, passim. BELLUTA, Hervè, Revoca o sostituzione di misura cautelare e limiti al coinvolgimento della vittima, in Dir. pen. cont., 28 novembre 2013, § 2.

41 Cfr. POTETTI, Domenico, Il nuovo art. 299 c.p.p. dopo il decreto legge n. 93 del 2013, in Cass. pen., 2014, p. 976.

42 VIGANÒ, Francesco, La tutela penale della libertà individuale, vol. I, L'offesa mediante violenza, Milano, 2002, p. 3.

43 Secondo VIGANÒ, Francesco, La tutela penale della libertà individuale, vol. I, L'offesa mediante violenza, Milano, 2002, p. 148-149, la violenza alle persone, diversamente da quella sulle cose sarebbe sempre caratterizzata da una «funzionalità coercitiva»; per SPAGNOLO, Paola, Vittima di reato e diritto all'informazione: un binomio insoddisfacente, in Cass. pen., 2017, p. 3488, nel concetto vanno ricomprese «tutte quelle forme di minaccia e/o intimidazione riconducibili alla violenza morale e psicologica». 
libertà di movimento del soggetto passivo ${ }^{44}$. Quest'ultima impostazione sarebbe dotata di una maggiore forza denotativa in grado di meglio delineare quali condotte criminali giustificano una tutela rafforzata delle vittime; al contempo però, rinuncerebbe a salvaguardare l'integrità psico-fisica di persone offese di reati quali i maltrattamenti in famiglia (art. 572 c.p.) o lo stalking (art. 612-bis c.p.) che spesso si manifestano con forme di violenza psicologica: negare in questi casi il diritto di conoscere l'eventuale provvedimento di favor libertatis emesso nei confronti del proprio persecutore esporrebbe la vittima al rischio di ripiombare nell'incubo, senza poter predisporre una qualche misura di protezione. Se interpretassimo in maniera riduttiva il concetto di violenza alla persona, le disposizioni a tutela delle vittime fallirebbero il loro obiettivo proprio rispetto a quei delitti che più di altri richiedono l'informazione necessaria a difendersi da una nuova vittimizzazione. Del resto, le indicazioni provenienti dalla normativa sovranazionale sembrano andare in questa direzione, adottando un concetto lato di violenza alla persona che ricomprenderebbe ogni atto in grado di provocare un danno fisico, emotivo, mentale, sessuale, psicologico e/o economico ${ }^{45}$.

Dunque, più utile ai nostri fini la prima delle teorie richiamate che consente di inglobare nel concetto di violenza alla persona anche la condotta strutturata in maniera tale da compromettere la libertà di determinazione della persona offesa ${ }^{46}$. $\mathrm{E}$ in questa direzione sembrano muoversi anche le sezioni unite della Corte di cassazione ${ }^{47}$ che, chiamate a pronunciarsi sul significato da attribuire all'omologa espressione contenuta

${ }^{44}$ V. BONTEMPELLI, Manfredi, Novità nelle procedure di revoca e sostituzione, in DIDDI, Alessandro - GERACI, Rosa Maria (a cura di), Misure cautelari ad personam in un triennio di riforme, Torino, 2015, p. 151 ss.; DIDDI, Alessandro, Chiaroscuri nella nuova disciplina sulla violenza di genere, in Proc. pen. giust., 2014, p. 99.

${ }_{45}$ Cfr. direttiva 2012/29/UE (considerato nn. 17 e 18); Convenzione di Istambul, art. 3; direttiva 2011/36/UE, art. 11, § 7.

46 In questo senso, POTETTI, Domenico, Il nuovo art. 299 c.p.p. dopo il decreto legge n. 93 del 2013, in Cass. pen., 2014, p. 980; dello stesso avviso, pure ZACCHÈ, Francesco, Il sistema cautelare a protezione della vittima, in BARGIS, Marta - BELLUTA, Hervè (a cura di), Vittime di reato e sistema penale. La ricerca di nuovi equilibri, Torino, 2017, p. 431.

47 Cass., s.u., 29 gennaio 2016, n. 10959, in Cass. pen., 2016, p. 2364 ss. Per un commento, v. AMOROSO, Maria Cristina, La nozione di delitti commessi con 
nell'art. 408 comma 3-bis c.p.p. ${ }^{48}$, hanno evidenziato che dalle convenzioni internazionali emergerebbe che la violenza alla persona viene sempre intesa in senso ampio, ricomprendendovi tanto le aggressioni fisiche che quelle psicologiche (ogni forma di violenza che possa manifestarsi nella relazione interpersonale ${ }^{49}$ : si tratterebbe, in ultima analisi, di un concetto di violenza assimilabile a quello di violenza di genere. Ciò non di meno, in quest'ultima affermazione può essere scorta la portata assai ridotta della decisione, la quale si è limitata a stabilire che nel concetto di delitti commessi con violenza alla persona rientrano sia i maltrattamenti in famiglia che lo stalking, senza provare a dare un significato di portata generale all'espressione. Anzi, l'approccio metodologico adottato è stato messo in discussione da altra giurisprudenza di legittimità, secondo la quale una corretta esegesi del testo normativo richiede la valorizzazione non tanto della fattispecie legale astratta connotata nel suo schema dogmatico dalla violenza alla persona, quanto piuttosto la sua manifestazione: se cioè sussista una condotta materiale caratterizzata dalla concreta esplicazione di atti di violenza in danno della persona offesa ${ }^{50}$. E questo consentirebbe di superare pure quell'indirizzo giurisprudenziale che àncora la nozione di «delitti commessi con violenza alla persona» all'esistenza di un pregresso rapporto tra vittima e reo, all'interno del quale si dovrebbero innestare episodi di violenza fisica o psicologica tali da far presumere l'esistenza del rischio di intimidazioni, ritorsioni o vittimizzazione secondaria ${ }^{51}$. In effetti, anche la vittima occasionale di delitti, consumati o tentati, che, in concreto, si sono manifestati con atti di violenza dovrebbe rientrare nel raggio di tutela

violenza alla persona: il primo passo delle Sezioni Unite verso un nuovo viaggio, in Cass. pen., 2016, p. 3714 ss.

48 Secondo Cass., sez. VI, 9 febbraio 2016, n. 6864, in Cass. pen., 2016, p. 3751 ss., il significato da attribuire alla locuzione «delitti commessi con violenza alla persona» è lo stesso tanto nel procedimento di archiviazione che in quello cautelare e impone all'autorità giudiziaria un onere rafforzato di informazione nei confronti della vittima.

49 In argomento, BRESSANELLI, Caty, La "violenza di genere" fa il suo ingresso nella giurisprudenza di legittimità: le Sezioni Unite chiariscono l'ambito di applicazione dell'art. 408 co. 3-bis c.p.p., in Dir. pen. cont., 21 giugno 2016.

${ }^{50}$ Cass., sez. I, 29 ottobre 2015, n. 49339, in Cass. pen., 2016, p. 4152. Nello stesso senso, Cass., sez. II, 24 giugno 2016, n. 30302, in Ced Cass., rv. 267718.

51 Cass., sez. II, 8 giugno 2017, n. 46996, in Proc. pen. giust., 2018, p. 305. 
della disciplina, per cui non risulterebbe conforme alla legge un'applicazione ristretta del dato normativo fondata sull'esistenza di un qualche legame tra i soggetti coinvolti, perché tradirebbe la ratio legis dell'introduzione di questi rafforzati meccanismi di informazione: salvaguardare le vittime di condotte violente «suscettibili di potenziale reiterazione in caso di modifica dello status cautelare del soggetto responsabile» ${ }^{52}$.

Certo, accogliere un concetto ampio di delitti commessi con violenza alla persona valorizza massimamente le istanze di protezione della vittima e combinato con un meccanismo comunicativo affidato all'autorità giudiziaria procedente non crea grandi contraccolpi sul sistema, in particolare sulla latitudine delle garanzie di cui gode la persona in vinculis; ad opposta conclusione si deve giungere, invece, quando ragioniamo dell'ampiezza dell'onere informativo nel procedimento per la revoca e la sostituzione di una misura cautelare, onere imposto all'istante ${ }^{53}$. In questo caso i contraccolpi sono rilevanti e in grado di nuocere al diritto del prevenuto ad una rivalutazione dello status custodiae.

\section{La duplice dimensione della comunicazione "RAFForzata" ALLA PERSONA OFFESA.}

Il corredo informativo pro victima si compone e si rafforza attraverso le disposizioni previste dagli artt. 282-quater e 299 commi 2-bis, 3 e 4-bis c.p.p. e opera su due livelli: uno speculare a quello dell'art. 90-ter c.p.p. che offre «una tutela minore e successiva (diritto di sapere)»; l'altro funzionale a consentire un inedito intervento della vittima nell'incidente cautelare (diritto di interloquire) ${ }^{54}$. Cominciamo dal primo che a sua volta contiene due distinte azioni comunicative.

Per quel che concerne la vittima ${ }^{55}$, l'art. 282-quater c.p.p., già con la rubrica, identifica in maniera chiara che si è in presenza, di un diritto

\footnotetext{
52 Ancora Cass., sez. I, 29 ottobre 2015, n. 49339, in Cass. pen., 2016, p. 4152.

53 V. infra, § 5 .

54 POTETTI, Domenico, Il nuovo art. 299 c.p.p. dopo il decreto legge n. 93 del 2013, in Cass. pen., 2014, p. 972.

55 La disposizione contiene anche oneri informativi in favore dell'accusato nei cui confronti sia stato disposto l'allontanamento dalla casa familiare o il
} 
alla comunicazione in merito all'adozione dei provvedimenti di cui agli artt. 282-bis e 282-ter c.p.p. (e soltanto in questi casi): una comunicazione postuma che agisce prevalentemente in funzione di attivazione di una tutela extra-processuale. Quando vengono adottate le misure cautelari dell'allontanamento dalla casa familiare e del divieto di avvicinarsi ai luoghi frequentati dalla persona offesa, "il protetto" viene informato delle prescrizioni che l'accusato deve osservare, mettendolo così nella condizione di sapere su quali margini di sicurezza può contare e, nel caso, segnalare eventuali trasgressioni ai fini di un possibile inasprimento della misura ${ }^{56}$. Peccato che l'obbligo di informativa non riguardi anche le altre cautele coercitive, in particolare divieto di dimora e arresti domiciliari, visto che le esigenze di protezione risultano superiori, quando per fronteggiare i pericula libertatis i dispositivi di cui agli artt. 282-bis e 282-ter c.p.p. sono stati ritenuti inefficaci ${ }^{57}$. Comunque, va detto che il raggio di azione della comunicazione è stato potenziato attraverso l'inserimento del comma 1-bis nell'art. 282-quater c.p.p., che prevede il diritto della vittima di essere informata della possibilità di estendere l'efficacia delle misure di protezione oltre i confini dello Stato che le ha adottate attraverso la richiesta dell'ordine di protezione europeo ${ }^{58}$.

Sulla stessa lunghezza d'onda, dal punto di vista degli scopi connessi all'informativa, sembra collocarsi l'art. 299 comma 2-bis c.p.p.

divieto di avvicinamento ai luoghi frequentati dalla persona offesa: nel caso in cui l'imputato si sia sottoposto positivamente a specifici programmi di prevenzione della violenza, il responsabile del servizio socio assistenziale che ha organizzato il programma, ne dà comunicazione al pubblico ministero ai fini della revoca o sostituzione in melius della misura. Si tratta di una disposizione che fa parte di quel sistema integrato di protezione delle vittime che ha tra i suoi punti di forza la prevenzione dei comportamenti violenti.

56 MORELLI, Francesco, Commento all'art. 9 d.l. 23 febbraio 2009, n. 11, in Legislaz. pen., 2009, p. 510.

57 Cfr., volendo, CARACENI, Lina, Misure cautelari pro victima e diritti di libertà dell'accusato: a proposito di una convivenza faticosa, in Arch. n. proc. pen., 2017, p. 254 ss.

58 Ai sensi dell'art. 2 comma 1, lett. $c$ d.lgs. 11 febbraio 2015 n. 9 infatti, la vittima può chiedere che gli effetti delle misure di protezione emesse dall'autorità giudiziaria di uno degli Stati membri dell'Unione Europea, si estendano al territorio di un altro paese membro nel quale la persona risieda o soggiorni o dichiari di voler risiedere o soggiornare. 
che prevede la comunicazione, sempre postuma, «a cura della polizia giudiziaria», dei provvedimenti di revoca o sostituzione in melius di una delle misure coercitive (ad eccezione del divieto di espatrio e dell'obbligo di presentazione alla polizia giudiziaria) adottate nei procedimenti aventi ad oggetto delitti commessi con violenza alla persona, onere informativo che deve essere assolto in favore della persona offesa e, ove nominato, del suo difensore, oltre ai servizi socio-assistenziali. Anche qui si tratta di una forma di comunicazione che mette la persona offesa in condizione di apprestare prevalentemente idonee strategie reattive, anche di natura extra-procedimentale, miranti alla sua protezione individuale, a fronte dello status libertatis riottenuto dal prevenuto ${ }^{59}$.

Non poche le incertezze interpretative che suscita una disposizione figlia della solita approssimazione della tecnica normativa, disattenta a qualsivoglia logica di sistema e persino alla coerenza interna della singola norma ${ }^{60}$. Innanzitutto, che si tratti di un "blando" meccanismo di tutela per la sicurezza della vittima (dunque un diritto minore) trova conferma nel fatto che nessuna sanzione processuale è stata prevista nel caso in cui la comunicazione non venga effettuata ${ }^{61}$, né può ricavarsi dal sistema delle invalidità: i diritti della persona offesa meritevoli di protezione, ai sensi dell'art. 178 lett. c c.p.p., sono soltanto quelli di partecipazione alla fase del giudizio ${ }^{62}$.

Pure l'ambito di applicazione dell'art. 299 comma 2-bis c.p.p., stando alla littera legis, sembra troppo circoscritto perché la disciplina assicuri una protezione effettiva: il congegno informativo che si attiva solo in caso di revoca o sostituzione della misura cautelare non tiene conto di tante altre situazioni di modifica pro libertate che, di conseguenza, non

59 In tal senso, anche CIAMPI, Stefano, Il diritto di difesa e all'informazione, in BARGIS, Marta - BELLUTA, Hervè (a cura di), Vittime di reato e sistema penale. La ricerca di nuovi equilibri, Torino, 2017, p. 293.

60 BELLUTA, Hervè, Revoca o sostituzione di misura cautelare e limiti al coinvolgimento della vittima, in Dir. pen. cont., 28 novembre 2013, § 4.

61 Diversamente da quanto accade, ad esempio, nell'ambito del procedimento di archiviazione: l'omessa notificazione della richiesta di archiviazione alla persona offesa determina la nullità del conseguente provvedimento (art. 410bis c.p.p.).

62 Sulla difficoltà di riconoscere alla vittima anche un diritto di impugnazione, v. infra, $\S 4$. 
verranno portate a conoscenza della vittima: basti solo pensare ai casi di estinzione automatica di una misura cautelare o alla sua applicazione con modalità meno gravose $\mathrm{e}^{63}$. Nel primo caso, può soccorrere l'art. 90ter c.p.p. ${ }^{64}$, mentre nel secondo lo sforzo di inglobare nella disciplina dell'art. 299 comma 2-bis c.p.p. anche le modifiche migliorative del regime cautelare imposto al prevenuto si deve a dottrina e giurisprudenza. Per non incorrere in una proliferazione ingiustificata di comunicazioni anche riguardanti cambiamenti davvero minimi nelle modalità esecutive, si è proposta una lettura adeguatrice, intendendosi per modifiche suscettibili di comunicazione quelle che determinano una «attenuazione sostanziale della restrizione della libertà personale ${ }^{65}$ che in qualche modo compromettano le istanze di protezione della vittima perché abbassano il livello di tutela ${ }^{66}$. Rilevano, in ultima analisi, quelle variazioni che possono ripristinare un contatto potenzialmente pericoloso tra accusato e "protetto", evenienza che il complessivo corredo di disposizioni informative tenderebbe a scongiurare.

Venendo al secondo dei livelli comunicativi introdotti in favore della vittima, l'art. 299 commi 3 e 4-bis c.p.p. descrive un vero e proprio diritto di conoscere l'evoluzione dello status libertatis del sottoposto alla coercizione per poter intervenire, attraverso la proposizione di memorie, nel procedimento di revoca instaurato a richiesta di parte, provando ad influire sul processo decisionale del giudice ${ }^{67}$. Nel primo caso si prevede che, nel corso delle indagini preliminari il pubblico ministero e la persona

63 Su tali aspetti, cfr. BONINI, Valentina, Il sistema di protezione della vittima e $i$ suoi riflessi sulla libertà personale, Padova, 2018, p. 371; SEPE, Giuseppe, Violenza di genere e consultazione della persona offesa nelle vicende estintive delle misure cautelari, in Dir. pen. cont., 9 luglio 2014, p. 5. Sul carattere parziale e deficitario della novella, v. VALENTINI, Elena, Il ruolo della persona offesa nella procedura di revoca e sostituzione delle misure cautelari personali, in CURI, Francesca (a cura di), Ordine pubblico e sicurezza nel governo della città, Bologna, 2016, p. 231. 
sottoposta alle indagini che chiedano la revoca o la sostituzione di una misura coercitiva nell'ambito di un procedimento per reati commessi con violenza alla persona (ad eccezione del divieto di espatrio e dell'obbligo di presentazione alla polizia giudiziaria) debbono, a pena di inammissibilità, notificare al difensore della persona offesa o, in mancanza di questo, alla vittima stessa (salvo che non abbia dichiarato o eletto domicilio) l'istanza di revoca o sostituzione della cautela. Fa eccezione l'istanza presentata in sede di interrogatorio di garanzia ${ }^{68}$.

Lo stesso incombente è imposto a pubblico ministero e imputato quando la richiesta di modifica viene proposta fuori udienza dopo la chiusura delle indagini preliminari. In tali evenienze siamo in presenza di una "informazione previa", necessaria per consentire alla vittima di attivarsi per scongiurare una soluzione pro libertate che determini una concreta riduzione della sua sfera di sicurezza: un protagonismo a difesa della propria incolumità che riscontriamo per la prima volta nel codice di rito penale; ed è anticipato da un vero e proprio diritto alla conoscenza (un diritto forte) presidiato da una inammissibilità della domanda di revoca o di sostituzione della misura ove la parte istante non assolva il dovere informativo.

Il carattere inedito dell'intervento della persona offesa non è tanto legato al contenuto delle prerogative processuali che le vengono riconosciute, quanto piuttosto al contesto nel quale possono essere esercitate: il procedimento cautelare, o meglio quel sub procedimento vocato a ripristinare la libertà dell'accusato quando siano venuti meno i presupposti che giustificavano la coercizione. Un diritto di interlocuzione che rischia di compromettere la funzionalità di questo incidente cautelare e di neutralizzarne la connotazione di garanzia, così spiccata da essere uno dei pochi dispositivi de libertate che può essere attivato pure ex officio (art. 299 comma 3, ult. parte c.p.p.). Dunque, per la prima volta l'offeso è contemplato «nella cognizione della fase esecutiva cautelare, tradizionalmente segnata dalla esclusiva contrapposizione dialettica tra autorità statale e imputato» ${ }^{69}$. Ed è

68 V. infra, § 4 .

69 GUERRA, Mariaemanuela, Questioni in tema di diritti di informazione ed interlocuzione della persona offesa nell'incidente cautelare, in Cass. pen., 2017, p. 2539. 
proprio la regolamentazione del meccanismo informativo preventivo in favore della vittima a mettere in discussione gli equilibri che sorreggono l'incidente cautelare, che carica sulle spalle del prevenuto il peso di un incombente quasi impossibile da assolvere a causa di una disciplina normativa approssimativa e piuttosto farraginosa, ma in grado di condizionare negativamente l'esito dell'istanza di revoca della misura che verrebbe condannata all'inammissibilità.

L'informativa dipendente dall'assolvimento di un incombente da parte dell'offeso: quello di nominare un difensore e dichiarare o eleggere domicilio per le istanze proposte fuori udienza: mancando questa espressione del suo interesse a partecipare al procedimento, sembrerebbe "saltare" nei commi 3 e 4-bis dell'art. 299 c.p.p. il dovere per il pubblico ministero, ma soprattutto per l'accusato, di notificare alla vittima la richiesta di revoca o sostituzione della misura. In definitiva, l'onere di informativa, da cui dipende l'ammissibilità della domanda di modificazione in melius dei lacci della coercizione, sorgerebbe soltanto quando la persona offesa dal reato possa essere raggiunta e messa in condizione di fornire il suo contributo alla decisione attraverso l'incarico dato ad un legale o una dichiarazione da cui si desuma l'individuazione di un domicilio (quantomeno di un luogo dove affermi di poter essere rintracciata) ${ }^{70} . \mathrm{E}$ poiché dall'informativa dipende la possibilità per l'imputato di vedere la sua istanza "presa in considerazione" dal giudice, si dovrà sempre accertare in concreto che l'accusato potesse accedere alle informazioni identificative della vittima in grado di metterlo nelle condizioni di assolvere al munus comunicativo ${ }^{71}$.

70 In questo senso, v. tra i molti, GUERRA, Mariaemanuela, Questioni in tema di diritti di informazione ed interlocuzione della persona offesa nell'incidente cautelare, in Cass. pen., 2017, p. 2551 ss., secondo la quale l'onere di notifica incombente specialmente sull'accusato è stato predisposto «al fine di realizzare una tutela proporzionata dei diritti della persona offesa alla partecipazione al procedimento che quello della persona indagata a sollecitare un tempestivo ripensamento della situazione cautelare che lo riguarda». In giurisprudenza, Cass., sez. II, 3 febbraio 2016, n. 12325, in Ced Cass., rv. 266435.

71 V. infra, § 5 . 


\section{Natura e ampiezza dell'interVento della Vittima Nel PROCEDIMENTO DI REVOCA DELLE MISURE.}

Gli obblighi di notificazione di cui all'art. 299 commi 3 e 4-bis c.p.p. sembrerebbero spianare la strada per il riconoscimento di un vero e proprio contraddittorio tra la vittima e il prevenuto in materia cautelare; in verità consentono di riconoscere all'offeso il più debole diritto di interlocuzione: benché questi non sia un mero destinatario di informazioni, la disciplina normativa richiamata non si spinge fino al punto da inserirlo tra i protagonisti di un autentico confronto dialettico. Il contributo che può offrire si riduce ad una memoria scritta, predisposta ai sensi dell'art. 121 c.p.p., cioè un atto a contenuto tipicamente argomentativo utile ad inserire nuovi elementi storico-fattuali ed eventuali considerazioni di ordine giuridico ${ }^{72}$, rimanendo tuttavia esclusa qualsiasi possibilità di avanzare richieste dirette all'organo giurisdizionale ${ }^{73}$. Dunque la vittima non partecipa al procedimento di revoca, se non come osservatore esterno interessato a far sentire le proprie ragioni ed esercitando una sorta di «facoltà di contraddire in anticipo» ${ }^{74} \mathrm{e}$, di conseguenza, non si può parlare di contraddittorio in senso proprio. Vi osterebbero due ulteriori ragioni non di poco momento: da un lato, il giudice, all'atto della decisione, non ha un obbligo di tenere conto degli argomenti addotti con la memoria, né la stessa vittima può impugnare il provvedimento di sostituzione o revoca ove quegli argomenti fossero stati ignorati ${ }^{75}$; dall'altro, il meccanismo

72 Sul punto, cfr. DALIA, Gaspare, I presupposti dell'obbligo di notifica alla persona offesa della richiesta di revoca o sostituzione della misura cautelare personale, in Proc. pen. giust., 2018, 2, p. 312.

73 Così, DI NUZZO, Viviana, Giudizio cautelare e tutela della persona offesa, tra istanze di protezione e prospettive di partecipazione attiva, in Legislaz. pen., 29.7.2020, p. 13.

74 BONTEMPELLI, Manfredi, Novità nelle procedure di revoca e sostituzione, in DIDDI, Alessandro - GERACI, Rosa Maria (a cura di), Misure cautelari ad personam in un triennio di riforme, Torino, 2015, p. 146.

75 L'impugnazione della persona offesa è esclusa tanto per il principio di tassatività in materia, quanto per la previsione dell'art. 572 c.p.p. secondo cui è il pubblico ministero a dover agire nell'interesse della persona offesa in tutti i casi in cui quest'ultima sia sprovvista di un potere di impugnazione diretta, come in questo caso. Cfr. ROMANELLI, Bartolomeo, Omessa notifica alla persona offesa della richiesta di revoca o sostituzione di misura cautelare 
dialettico risulta imperfetto pure sul fronte della difesa dell'imputato, poiché la decisione del giudice interviene senza che questa abbia avuto modo di conoscere gli (e di interloquire sugli) elementi probatori addotti dalla vittima ${ }^{76}$.

Vieppiù, questo pseudo "contraddittorio cartolare" è stato tradotto in disposizioni così confuse nel lessico impiegato e sistematicamente spurie da suscitare più di una questione esegetica in grado di contenerne (se non neutralizzarne) ogni efficacia. L'art. 299 comma 3 c.p.p. riconosce un potere concorrente a offeso e difensore (testimoniato dall'uso della congiuntiva «e») di presentare memorie nei due giorni successivi alla notifica dell'istanza di revoca o sostituzione della misura, una previsione che devia dai poteri classici di cui all'art. 90 c.p.p. che attribuisce detta facoltà direttamente alla persona offesa. Forse sarebbe bastato un richiamo a quest'ultima disposizione, anche se la diversa formulazione potrebbe essere giustificata dall'intento di dare maggior forza alla previsione, magari configurando apporti diversi da parte dei due soggetti ${ }^{77}$. In realtà, nel complesso, si rivela incapace di offrire ogni contributo utile, attesi i tempi "strettissimi" in cui la memoria va presentata (due giorni dalla

coercitiva: problemi definitori e rimedi in sede di impugnazione, in Cass. pen., 2017, p. 4437, secondo il quale nemmeno ricorrendo alla previsione di cui all'art. 568 comma 2 c.p.p. sarebbe possibile ritiene alla persona offesa un diritto all'impugnazione, come invece riconosce parte della giurisprudenza (Cass., sez. VI, 9 febbraio 2016, n. 6864, in Cass. pen., 2016, p. 3751; Cass., sez. V, 20 settembre 2016, n. 7404, in Cass. pen., 2017, p. 4426); l'art. 568 comma 2 c.p.p. fissa l'impugnabilità oggettiva di tutti i provvedimenti riguardanti la libertà personale, se non altrimenti impugnabili, ma anche questa norma richiede un «coordinamento con il principio di tassatività, sancito, sotto il profilo soggettivo, dall'art. 568 comma 3 c.p.p. [...] posto che la legittimazione ad impugnare della persona offesa è limitata alle sole ipotesi riconosciute dalla legge, il ricorso all'art. 568 comma 2 c.p.p. non può valere a colmare la lacuna dell'art. 299 c.p.p.». Esclude il potere di impugnazione della persona offesa, Cass., sez. V, 17 maggio 2017, n. 54319, in Cass. pen., 2018, p. 2591.

76 Cfr., ZACCHÈ, Francesco, Il sistema cautelare a protezione della vittima, in BARGIS, Marta - BELLUTA, Hervè (a cura di), Vittime di reato e sistema penale. La ricerca di nuovi equilibri, Torino, 2017, p. 435.

77 Ciò che ci si aspetta dalla vittima è l'introduzione di elementi fattuali, circostanze, sopravvenienze tali da ampliare il quadro conoscitivo del giudice e "sconsigliare" una riforma in melius, mentre il legale potrebbe offrire argomentazioni tecnico-giuridiche miranti ad ottenere lo stesso risultato. 
notificazione della richiesta di revoca). Del resto, sull'idoneità di questa scansione temporale ad assicurare l'intervento della persona offesa è lecito nutrire più di un dubbio, atteso che nessuna disposizione impedisce al giudice di decidere prima della scadenza dei due giorni per il deposito della memoria dell'offeso, né conseguenze sanzionatorie possono essere ricavate dal sistema ${ }^{78}$, a differenza di ciò che accade per la speculare facoltà riconosciuta al pubblico ministero di presentare un parere: ai sensi dell'art. 299 comma 3-bis c.p.p. il giudice, prima di decidere sulla revoca o sulla sostituzione sente il pubblico ministero, anche se il suo parere non è vincolante. Il mancato rispetto di detto termine può essere ricondotto all'art. 178 lett. $b$ c.p.p. come violazione di una disposizione che assicura il diritto della parte pubblica di partecipare al procedimento (nullità a regime intermedio ex art. 180 c.p.p.).

Sempre dal punto di vista dell'effettività del diritto di intervento della persona offesa, ancor più grave si rivela la scelta legislativa di ricorrere a disposizioni dal diverso tenore nei commi 3 e 4-bis dell'art. 299 c.p.p.: la facoltà di presentare memorie ex art. 121 c.p.p., stando alla lettera della legge, è espressamente riconosciuta solo nel caso di istanza di revoca presentata nel corso delle indagini preliminari, mentre se questa è proposta una volta chiusa la fase investigativa, la vittima avrebbe solo diritto all'informativa e non anche all'interlocuzione ${ }^{79}$. Differenza davvero poco ragionevole che può essere recuperata ritenendo applicabile la disciplina generale dell'art. 90 c.p.p.: l'offeso non avrebbe un termine imposto per presentare la memoria e correrebbe solo il rischio che il suo contributo arrivi a decisione già assunta. Ma si tratterebbe davvero di una differenza minima tra i due regimi, atteso che, come abbiamo visto, anche nell'ipotesi del comma 3 dell'art. 299 c.p.p. il termine è sprovvisto di sanzione.

78 DIDDI, Alessandro, Chiaroscuri nella nuova disciplina sulla violenza di genere, in Proc. pen. giust., 2014, p.100. Nello stesso senso, PROCACCINO, Angela, L'avvento della persona offesa nelle dinamiche custodiali, in DIDDI, Alessandro - GERACI, Rosa Maria (a cura di), Misure cautelari ad personam in un triennio di riforme, Torino, 2015, p. 100. V. anche supra, § 3.

79 POTETTI, Domenico, Il nuovo art. 299 c.p.p. dopo il decreto legge n. 93 del 2013, in Cass. pen., 2014, p. 985. 
L'approssimativa formulazione del comma 4-bis dell'art. 299 c.p.p. non rende agevole nemmeno determinare se il dovere informativo imposto all'imputato, una volta chiuse le indagini preliminari, riguardi tanto le istanze presentate fuori udienza che quelle proposte in udienza ${ }^{80}$ : se lo scopo della disciplina è quello di mettere in condizione l'offeso di fornire il proprio contributo nella procedura, il bisogno di una notificazione potrebbe sussistere anche per quelle istanze di modifica del regime cautelare presentate in udienza quando la vittima non sia presente (né come offeso, né come parte civile), se non vi sono altri modi legittimi e sicuri attraverso i quali possa venirne effettivamente a conoscenza ${ }^{81}$.

Non meno problematica si prospetta la questione dell'intervento della vittima nel procedimento di revoca o sostituzione di una misura cautelare nel caso in cui venga attivato ex officio, ai sensi dell'art. 299 comma 3 c.p.p. Un'informativa non è prevista e, di conseguenza, porterebbe a concludere che la decisione debba fare a meno del contributo dell'offeso, con evidenti profili di illegittimità costituzionale ex art. 3 Cost., atteso che se la disciplina intende salvaguardare l'interesse della persona offesa a conoscere eventuali modifiche in melius dello status

80 Fornisce una lettura restrittiva Cass., sez. V, 8 novembre 2018, n. 9872, in Dir. pen cont., 24 giugno 2019, rilevando l'inutilità dell'onere informativo per la parte istante in tutti i casi in cui l'offeso, presente all'udienza, è in condizione di conoscere l'avvio della procedura cautelare.

81 Di diverso avviso BONTEMPELLI, Manfredi, Novità nelle procedure di revoca e sostituzione, in DIDDI, Alessandro - GERACI, Rosa Maria (a cura di), Misure cautelari ad personam in un triennio di riforme, Torino, 2015, p. 162, secondo il quale l'assenza dell'offeso sarebbe sintomatica del suo "disinteresse" per le sorti della vicenda cautelare, che avrebbe potuto monitorare attraverso l'intervento o la costituzione di parte civile. L'A. suggerisce di "trattare" l'offeso alla stessa stregua del pubblico ministero, il quale se l'istanza è proposta in udienza, non ha diritto di ricevere la comunicazione ai fini della proposizione del proprio parere. In verità, il p.m. è una delle parti necessarie del processo e conseguentemente sempre sarà a conoscenza in udienza dell'istanza di revoca/sostituzione di una misura cautelare, a differenza delle altre parti private diverse dall'imputato e dell'offeso che solo eventualmente sono presenti durante il processo (tanto nell'udienza preliminare che nel giudizio vero e proprio). E a giustificare l'assenza vi possono essere tante ragioni che non legittimano un superamento dell'onere informativo; paradossalmente, a tenere lontano l'offeso dal processo potrebbero essere proprio i rischi di vittimizzazione reiterata $\mathrm{o}$, in generale, quel diritto all'oblio che pure gli deve essere riconosciuto. 
custodiae dell'accusato per poter interagire nel processo decisionale, questo prescinde da chi assume l'iniziativa della modifica ${ }^{82}$. E malgrado sia stata fornita una lettura costituzionalmente orientata volta ad estendere sin d'ora l'onere informativo anche ai casi di revoca officiosa ${ }^{83}$, qualunque sforzo esegetico appare frustrato dal dato testuale che non consente appigli validi per superare la lacuna; solo una declaratoria di incostituzionalità o un intervento normativo sarebbero risolutori.

Infine, l'ambito oggettivo di incidenza dell'interlocuzione dell'offeso risulta ancor più circoscritto se si guarda a quali istanze impongono un onere informativo verso la vittima: a rigore la formulazione della norma prevede la notificazione delle sole richieste di revoca e sostituzione della misura cautelare, non anche dell'applicazione della misura con modalità meno gravose e perciò il contraddittorio cartolare dovrebbe essere escluso, malgrado vi possano essere dei cambiamenti nel regime esecutivo tali da generare timori e pericoli concreti per la vittima al pari di una revoca o di una sostituzione ${ }^{84}$.

Quel che è certo è che la notificazione non è dovuta nei casi in cui la richiesta di revoca è formulata in sede di interrogatorio ex art. 294 c.p.p., come stabilisce l'art. 299 comma 3 c.p.p.: qui prevalgono le istanze di tutela del diritto alla libertà dell'accusato che per la prima volta dalla privazione della libertà entra in contatto con il giudice ${ }^{85}$ ed esprime il momento massimo di garanzia per l'imputato nel procedimento cautelare.

82 Del resto, non tutte le ipotesi di revoca officiosa (proroga delle indagini, incidente probatorio, interrogatorio di garanzia, udienza preliminare e giudizio) consentono alla persona offesa di essere comunque informata e conseguentemente di intervenire presentando memorie ai sensi dell'art. 90 comma 1 c.p.p. POTETTI, Domenico, Il nuovo art. 299 c.p.p. dopo il decreto legge n. 93 del 2013, in Cass. pen., 2014, p. 984.

84 Sul punto, ancora BONTEMPELLI, Manfredi, Novità nelle procedure di revoca e sostituzione, in DIDDI, Alessandro - GERACI, Rosa Maria (a cura di), Misure cautelari ad personam in un triennio di riforme, Torino, 2015, p. 162. Di segno opposto le conclusioni di MARCHETTI, Filippo, L'intervento dell'offeso nel procedimento di revoca e sostituzione di una misura cautelare personale nuovamente sotto la lente della Suprema Corte, in Dir. pen. cont. 2019, 6, p. 128.

85 PROCACCINO, Angela, L'avvento della persona offesa nelle dinamiche custodiali, in DIDDI, Alessandro - GERACI, Rosa Maria (a cura di), Misure cautelari ad personam in un triennio di riforme, Torino, 2015, p. 97. 


\section{I RIFLESSI DELLA DISCIPLINA SUI DIRITTI DI LIBERTÀ DEL SOGGETTO IN VINCULIS.}

Gli approdi esegetici fin qui attinti per dare effettività a quella nuova dimensione dialettica che contrappone la vittima all'offensore nel contesto processuale de libertate necessitano ora di una "prova di resistenza", calati in quegli snodi del rito cautelare in cui più delicata si fa l'opera di bilanciamento tra opposti interessi per il più elevato livello di garanzie che circonda i diritti di libertà dell'accusato.

E partiamo dal punto critico dell'intero impianto normativo: la complessa attività informativa preparatoria all'intervento della vittima nel procedimento di revoca di una misura cautelare è stata interamente posta sulle spalle del soggetto in vinculis, con una pesante sanzione (l'inammissibilità dell'istanza) ove non venga compiutamente assolta e senza mettere in condizioni il prevenuto di esercitare il suo munus, causa una littera legis dai contorni imprecisi e sfumati. E il paradosso finale, che chiude il cerchio dell'irragionevolezza della disciplina, si ha quando è il pubblico ministero a dover comunicare l'avvio del sub procedimento ex art. 299 c.p.p.: una violazione processuale allo stesso imputabile determina l'inammissibilità dell'istanza e ricadute sullo status libertatis del prevenuto ${ }^{86}$. Il procedimento di revoca è la massima espressione del diritto all'habeas corpus: dunque è già discutibile che possa essere compromesso da un onere informativo verso la vittima gravante sullo stesso accusato; figuriamoci quando sia condizionato da un inadempimento imputabile al pubblico ministero. Del resto, è già a dir poco discutibile far dipendere i diritti di informazione e di partecipazione dell'offeso dalle scelte difensive messe in campo dall'accusato. Così, il dispositivo normativo congegnato realizza una protezione insoddisfacente dei bisogni conoscitive della vittima rispetto alle vicende cautelari, nell'aspettativa di "mettere al sicuro" la propria persona $^{87}$ e attua al contempo «un bilanciamento claudicante con il

\footnotetext{
86 BONTEMPELLI, Manfredi, Novità nelle procedure di revoca e sostituzione, in DIDDI, Alessandro - GERACI, Rosa Maria (a cura di), Misure cautelari ad personam in un triennio di riforme, Torino, 2015, p. 155.

87 V. supra, § 3 e 4.
} 
diritto di difesa dell'indagato, gravato da adempimenti irragionevoli, ancor meno ragionevolmente sanzionati» ${ }^{88}$.

Il mal riuscito contemperamento degli opposti interessi già risulta dall'individuazione dell'ambito applicativo della disciplina: quei «delitti commessi con violenza alla persona» in grado di condizionare la procedibilità dell'istanza de libertate e quindi - di fatto - l'esercizio del diritto di difesa da parte dell'imputato e l'interesse di questi a non vedere ingiustificatamente negato o sospeso l'esame della richiesta di revoca ${ }^{89}$. Il tentativo che giurisprudenza e dottrina hanno fatto di delineare la portata dell'espressione nell'art. 299 c.p.p. non ha raggiunto un livello appagante. In analogia con quanto visto a proposito del diritto all'informazione di cui all'art. 90-ter c.p.p. ${ }^{90}$, pure in questo contesto si fronteggiano i due orientamenti divergenti già evidenziati: una lettura più rigorosa ritiene che vi sia un diritto della vittima di interlocuzione nella vicenda cautelare soltanto in caso di condotta violenta in danno di una persona “mirata”, perché maturata nell'ambito di preesistenti relazioni personali"1; un'interpretazione più ampia ricostruisce il concetto di «delitti commessi con violenza alla persona» come categoria di portata generale - a partire dall'art. 12 preleggi - che non prevede quale criterio aggiuntivo per la sua delimitazione l'esistenza di un vincolo relazionale-affettivo tra presunto autore e vittima ${ }^{92}$.

88 CATALANO, Elena, La tutela della vittima nella direttiva 2012/29/UE e nella giurisprudenza delle Corti europee, in Riv. it. dir. proc. pen., 2014, p. 1804. Cass., sez. II, 12 ottobre 2017, n. 46996, in Proc. pen. e giust., 2018, 2, p. 305 ss. V. supra, § 2 .

91 BELLUTA, Hervè, Revoca o sostituzione di misura cautelare e limiti al coinvolgimento della vittima, in Dir. pen. cont., 28 novembre $2013, \S 3$, secondo il quale la norma "aperta" merita «una lettura a stretto rigore interpretativo, ma non legittima una scelta sul tipo di condotta violenta praticata in concreto». In giurisprudenza, v. Cass., sez. II, 14 ottobre 2015, n. 43353, in Ced Cass., rv. 265094 e Cass., sez. II, 25 maggio 2016, n. 25135, in Ced Cass., rv 267236.

92 Propendono per questa impostazione: RECCHIONE, Sandra, La vittima cambia il volto del processo penale: le tre parti "eventuali", la testimonianza dell'offeso vulnerabile, la mutazione del principio di oralità, in Dir. pen. cont., 16 gennaio 2017, p. 8; BONTEMPELLI, Manfredi, Novità nelle procedure di revoca e sostituzione, in DIDDI, Alessandro - GERACI, Rosa Maria (a cura di), Misure cautelari ad personam in un triennio di riforme, Torino, 2015, p. 151. 
Quanto al primo dei due orientamenti, se lo scopo dell'informativa di cui ai commi 3 e 4-bis dell'art. 299 c.p.p. è quello di mettere in condizione l'offeso di prendere parte al "contraddittorio cartolare" adducendo, attraverso memorie difensive, gli elementi ulteriori che giustificano il mantenimento del vincolo custodiale sull'accusato, vincolo teso a scongiurare una reiterazione della violenza, soltanto la preesistenza di un legame tra i due antagonisti può consentire questa incursione: difficile immaginare un contributo probatorio della "vittima occasionale" per impedire una modifica pro libertate ${ }^{93}$. Per converso, la seconda impostazione sembra meglio attuare gli intendimenti ricavabili dalla direttiva 2012/29/UE che vuole assicurare un'effettiva partecipazione al procedimento a tutte le vittime, identificate attraverso il pericolo o il rischio concreto di danno ${ }^{94}$.

Tuttavia, se quest'ultima lettura appare più convincente nell'ottica di un rafforzamento delle possibilità di protezione della vittima, non soddisfa dal punto di vista del funzionamento del meccanismo informativo introdotto nell'art. 299 commi 3 e 4-bis c.p.p. sul versante dei diritti difensivi dell'accusato. Infatti, se un'esegesi rigorosa del concetto di «violenza alla persona» circoscrive la tutela rafforzata alle sole vittime di violenza fisica e psicologica nell'ambito delle relazioni interpersonali, lasciando fuori un'ampia fetta di soggetti meritevoli di protezione, per contro, una interpretazione "espansiva" lo esporrebbe alla perdita di ogni capacità denotativa e chiamerebbe l'istante al difficile (se non impossibile) compito di determinare quando il reato possa dirsi commesso con violenza

93 GUERRA Mariaemanuela, Questioni in tema di diritti di informazione ed interlocuzione della persona offesa nell'incidente cautelare, in Cass. pen., 2017, p. 2546. Secondo l'A., pure la disposizione di cui all'art. 299 comma 2-bis c.p.p. sembra legittimare questa lettura restrittiva del concetto di violenza alla persona, poiché imponendo un onere comunicativo relativo alla revoca o alla sostituzione in melius delle misure cautelari coercitive verso i servizi socio-assistenziali e il difensore dello stesso offeso (in mancanza di quest'ultimo all'offeso stesso), prefigura una vittima ben determinata, che necessita di un'opera di aiuto e sostegno, bisogno riscontrabile generalmente nei reati di matrice domestica o "di genere" che presuppongono dunque una relazione tra autore e vittima.

94 Per una ricognizione della giurisprudenza sul tema, prevalentemente orientata per una interpretazione estensiva, v. BONINI, Valentina, Il sistema di protezione della vittima e i suoi riflessi sulla libertà personale, Padova, 2018, p. 385. 
alla persona, valutazione da cui dipende il suo diritto all'habeas corpus. Anche l'individuazione degli "incidenti cautelari" che impongono al prevenuto un dovere informativo non è così scontata. Dalla formulazione dell'art. 299 commi 3 e 4-bis c.p.p. vi è chi ha ritenuto che pure la modifica delle modalità esecutive della misura obblighi alla notificazione dell'istanza alla persona offesa ${ }^{95}$, mentre altri rifiutano questa impostazione, ritenendo che il legislatore abbia inteso non "appesantire troppo" il sub procedimento, «rimettendo al giudice le valutazioni del caso, anche finalizzate alla tutela della persona offesa ${ }^{96}$. E quand'anche l'imputato riuscisse a sciogliere il dubbio sull'an dell'informativa, non pochi problemi si troverebbe ad affrontare con riferimento al quomodo.

Innanzitutto la legge impone al comma 3 dell'art. 299 c.p.p. che la notificazione della richiesta di revoca o sostituzione della misura alla persona offesa sia «contestuale» alla presentazione dell'istanza, il che dovrebbe obbligare il richiedente alla notificazione prima ancora del deposito ${ }^{97}$, anche se una notificazione fatta successivamente non dovrebbe invalidare l'istanza, la quale diverrebbe inammissibile soltanto nel caso in cui la notificazione non venisse effettuata ${ }^{98}$. Inoltre, l'onere è legato alla

95 BELLUTA, Hervè, Revoca o sostituzione di misura cautelare e limiti al coinvolgimento della vittima, in Dir. pen. cont., 28 novembre 2013, § 2. Per ulteriori approfondimenti, v. anche supra, § 4.

BONTEMPELLI, Manfredi, Novità nelle procedure di revoca e sostituzione, in DIDDI, Alessandro - GERACI, Rosa Maria (a cura di), Misure cautelari ad personam in un triennio di riforme, Torino, 2015, p. 162; POTETTI Domenico, Il nuovo art. 299 c.p.p. dopo il decreto legge n. 93 del 2013, in Cass. pen., 2014, p. 975. La giurisprudenza si è espressa negando un diritto all'informazione in un caso in cui il cambiamento delle modalità esecutive della misura (nel caso di specie obbligo di dimora con obbligo di presentazione alla polizia giudiziaria) si era tradotta, per il giudice, in un aumento di garanzie e tutela della persona offesa: Cass., 6 luglio 2016, n. 27975, in Ced Cass., n. 267131. BONTEMPELLI, Manfredi, Novità nelle procedure di revoca e sostituzione, in DIDDI, Alessandro - GERACI, Rosa Maria (a cura di), Misure cautelari ad personam in un triennio di riforme, Torino, 2015, p. 149, nonché POTETTI, Domenico, Il nuovo art. 299 c.p.p. dopo il decreto legge n. 93 del 2013, in Cass. pen., 2014, p. 982.

98 La contestualità di istanza e notificazione all'offeso dovrebbe essere assicurata (e conseguentemente l'ammissibilità della domanda di revoca/sostituzione) dal deposito insieme alla richiesta di revoca della prova dell'avvenuta notificazione. 
nomina di un difensore da parte della vittima o all'individuazione di un domicilio dove può essere raggiunta. E non sempre è facile per l'imputato entrare in possesso di queste informazioni, soprattutto quando l'incidente cautelare si innesta in una fase molto precoce del procedimento penale, caratterizzata da un elevato tasso di segretezza ${ }^{99}$. Invero, oltre all'evenienza in cui la vittima non fornisce le indicazioni necessarie alla notifica, vanno presi in considerazione tutti i casi in cui, soprattutto nel corso delle indagini, non siano conoscibili gli atti che consentono l'identificazione necessaria, o la vittima sia protetta e tenuta a "debita distanza" dal presunto aggressore; qui l'impossibilità di acquisire i dati necessari non può essere in alcun modo addebitata all'accusato e pregiudicare il suo diritto ad una rapida ed effettiva rivalutazione dello status libertatis ${ }^{100}$. Infine, la littera legis sul punto non tiene conto dei desiderata di quelle vittime che, una volta avviata la macchina processuale, sono refrattarie ad ogni successivo coinvolgimento ${ }^{101}$.

Una così complessa e defatigante attività preparatoria alla decisione in materia di revoca potrebbe presentare dubbi di compatibilità con il principio della ragionevole durata del processo che opera anche in materia cautelare ${ }^{102}$. Invero, la garanzia della durata ragionevole consente il ricorso a dispendiose articolazioni procedurali solo laddove esse siano finalizzate alla difesa di interessi meritevoli di protezione, nel ragionevole bilanciamento con altri valori concorrenti. Nel caso di specie, il diritto della vittima di essere informata non gode di una chiara

99 Anche per l'imputato in possesso di adeguata assistenza tecnico-difensiva sarebbe un'attività particolarmente complicata, figuriamoci per chi è privo di difensore o al massimo può contare su quello d'ufficio. Cfr. SEPE, Giuseppe, Violenza di genere e consultazione della persona offesa nelle vicende estintive delle misure cautelari, in Dir. pen. cont., 9 luglio 2014, p. 2

${ }^{100}$ In questo senso, Cass., sez. II, 12 ottobre 2017, n. 46996, in Proc. pen. giust., 2018, f. 2, p. 305 ss.

${ }^{101}$ RIZZATO, Emma, L. 119/13, le novità in materia di contrasto al c.d. femminicidio, in Quest. giust., 28 ottobre 2013. Anche per la vittima destinataria di un ordine di protezione che la colloca in un luogo segreto dovrebbe essere escluso l'obbligo di notificazione nel caso in cui non abbia provveduto alla nomina di un difensore.

${ }^{102} \mathrm{Su}$ questo aspetto, intendendo il canone dello speedy trial in chiave di tutela dell'imputato, VICOLI, Daniele, La "ragionevole durata" delle indagini, Torino, 2012, p. 57 ss., spec. 101. 
copertura costituzionale equiparabile a quella propria delle garanzie difensive e della libertà personale. Conseguentemente, ove sia difficile o impossibile "raggiungere" l'offeso dal reato, il suo interesse ad una "conoscenza legale" della richiesta di revoca di una misura cautelare «non sembra meritevole di essere protetto, a scapito dell'interesse ad una decisione tempestiva sulla libertà personale dell'imputato» ${ }^{103}$. Leggere diversamente la normativa, come pure fa parte della dottrina e della giurisprudenza, prevedendo la notificazione all'offeso anche in mancanza di una elezione di domicilio ${ }^{104}$, significherebbe gravare l'accusato di un dovere quasi impossibile da assolvere ${ }^{105}$. C'è da augurarsi che con la recente ordinanza di rimessione alle sezioni unite ${ }^{106}$ la Corte di Cassazione si orienti per una esegesi restrittiva che, dando rilievo alle scelte operate dalla vittima ${ }^{107}$, circoscriva i doveri informativi imposti all'accusato da cui, peraltro, non dovrebbe mai dipendere il coinvolgimento dell'offeso nel procedimento cautelare. D'altro canto, il deficit di determinatezza delle norme introdotte che non permette di fissare il «discrimen tra le ipotesi in cui il richiedente deve coinvolgere l'offeso e quelle in cui la "partita"

${ }^{103}$ BONTEMPELLI, Manfredi, Novità nelle procedure di revoca e sostituzione, in DIDDI, Alessandro - GERACI, Rosa Maria (a cura di), Misure cautelari ad personam in un triennio di riforme, Torino, 2015, p. 157, secondo il quale un razionale bilanciamento degli interessi in gioco si avrebbe nel momento in cui il dovere di notificazione fosse legato alla scelta dell'offeso di nominare un difensore o comunque di eleggere domicilio in mancanza di un legale, quindi dovere sì, ma quando si siano create le condizioni per una celere procedura connessa ad una certa reperibilità del destinatario della comunicazione.

104 POTETTI, Domenico, Il nuovo art. 299 c.p.p. dopo il decreto legge n. 93 del 2013, in Cass. pen., 2014, p. 986; in giurisprudenza, Cass., sez. II, $1^{\circ}$ aprile 2016, n. 19704, in Ced Cass., n. 267295; Cass., sez. III, 21 luglio 2020, n. 31191, in Ced Cass., n. 280363; Cass., sez II, 10 febbraio 2021, n. 12377, in Ced Cass., n. 280999.

105 Tra i molti, SEPE, Giuseppe, Violenza di genere e consultazione della persona offesa nelle vicende estintive delle misure cautelari, in Dir. pen. cont., 9 luglio 2014 , p. 2. Non sembra condividere queste conclusioni Cass., sez. II, 23 aprile 2020, n. 12800, in www.processopenaleegiustizia.it, la quale ha evidenziato che l'allungamento delle tempistiche necessarie per la statuizione sulla cautela sarebbe giustificato dal fatto che il procedimento cautelare si conclude con decisioni rilevanti per l'incolumità della vittima.

${ }^{106}$ Cass., sez. I, ord. 4 maggio 2021, n. 22444, in Sistema pen., 11 giugno 2021.

107 In questo senso, cfr. Cass., sez. I, 17 gennaio 2020, n. 5552, in Ced. Cass., rv. 278483; Cass., sez. I, 24 novembre 2020, n. 1460, in Ced Cass., rv. 280219. 
cautelare si gioca tra i consueti attori» ${ }^{108}$ impone una interpretazione di stretta legalità, per riportare la disciplina nella cornice dell'art. 13 Cost. che governa la materia de libertate $e^{109}$.

\section{ConClusioni: PROSPETTIVE DI RIFORMA.}

La strada aperta dalla direttiva 2012/29/UE prefigura un nuovo protagonismo processuale per la vittima che diventa «fattore di umanizzazione del diritto penale, materiale per la costruzione di un processo penale antropocentrico» ${ }^{110}$ che si articola secondo canoni attenti alla tutela dei diritti di tutti i soggetti coinvolti. Tuttavia, si tratta di uno scenario ancora di là da venire, almeno nell'ordinamento italiano: gli interventi fin ora messi in atto sono stati espressione di una visione riduttiva che mostra sempre la vittima come un soggetto da proteggere piuttosto che un co-protagonista in grado di entrare nell'agone dialettico proponendo le proprie istanze al giudice, così da incidere direttamente sulle sorti del procedimento. Vi è ancora una certa ritrosia «a concepire la vittima come titolare di autonomi poteri capaci di incidere attivamente sulle dinamiche cognitive, e conseguentemente, di condizionare gli epiloghi processuali» ${ }^{111}$, anche se il terreno ideale per questa diversa dimensione

${ }^{108}$ BONINI, Valentina, Il sistema di protezione della vittima e i suoi riflessi sulla libertà personale, Padova, 2018, p. 380. Per un approfondimento, v. sub § 6 .

109 VALENTINI, Elena, Il ruolo della persona offesa nella procedura di revoca e sostituzione delle misure cautelari personali, in CURI, Francesca (a cura di), Ordine pubblico e sicurezza nel governo della città, Bologna, 2016, p. 233.

${ }^{110}$ CATALANO, Elena, La tutela della vittima nella direttiva 2012/29/UE e nella giurisprudenza delle Corti europee, in Riv. it. dir. proc. pen., 2014, p. 1800.

${ }^{111}$ Per questo diverso approccio nella definizione del ruolo della vittima nel rito penale, PAULESU, Pier Paolo, Vittima del reato e processo penale: uno sguardo d'insieme (informazioni, diritti, tutele), in BARGIS, Marta - BELLUTA, Hervè (a cura di), Vittime di reato e sistema penale. La ricerca di nuovi equilibri, Torino, 2017, p. 127 ss., secondo il quale quello della vittima è ancora un ruolo servente, in particolare rispetto alle esigenze cognitive del processo: contano poco le sue aspettative in termini di esiti processuali, mentre contano tanto le informazioni che può fornire al giudice. Come precisato da CORDERO, Franco, Procedura penale, Torino, 2012, p. 277, la vittima, pur portatrice di autonomi interessi rispetto agli esiti del procedimento, resta «subalterna al pubblico ministero: gli apporta lumi; lo stimola 
partecipativa non è certamente il sistema de libertate, dove l'intervento giurisdizionale è congegnato in modo tale da difendere prioritariamente i diritti di libertà dell'accusato.

Senza dubbio la vittima non può più essere esclusa dalle dinamiche dell'accertamento penale, nemmeno da quelle cautelari, ma vanno comunque arginate derive "vittimocentriche", ossia quelle che, in nome della protezione delle vittime, sacrificano i diritti dell'imputato. Si dovrebbe non tanto costruire un muro di cinta intorno al processo, ma aprire dei varchi sicuri in cui chi ha subito le conseguenze del reato possa entrare non più come solo destinatario di interventi "a protezione" decisi e azionati da altri, ma come colui che «contribuisce attivamente alla funzione cognitiva del processo» ${ }^{112}$. Si tratta di una non facile operazione di contemperamento di interessi antitetici che si fa particolarmente delicata nel rito cautelare: essa richiede scelte normative chiare, decise e razionali (quelle che sono mancate sin'ora), all'interno delle quali situare l'intervento discrezionale del giudice che, «in ogni singolo caso ove si riscontra un pericolo di compromissione dei diritti dell'imputato, dovrebbe "mediare" tra esigenze difensive di quest'ultimo e necessità di tutela dell'offeso» ${ }^{113}$. Perché, nel rispetto del principio di legalità e di coerente e uniforme applicazione della legge, la ricerca di un tale equilibrio andrebbe fatta sul piano normativo, così da ridurre l'insorgenza di potenziali contrasti per le scelte operate dall'interprete ${ }^{114}$.

a mosse istruttorie o ad impugnare; e ogni rifiuto rivoltole deve essere motivato, senza, però, diritto di replica».

${ }^{112}$ In questo senso, SPAGNOLO, Paola, Vittima di reato e diritto all'informazione: un binomio insoddisfacente, in Cass. pen., 2017, p. 3490.

113 VENTUROLI, Marco, La vittima nel sistema penale: dall'oblio al protagonismo?, Jovene, 2015, pp. 119-120.

${ }^{114}$ Si tratta di uno schema decisorio analogo a quell'individual assessment proposto dalla direttiva 2012/29/UE, un modello normativo nella cui cornice valutare la vulnerabilità delle vittime nel caso concreto e adottare le adeguate misure di sostegno e protezione funzionali a scongiurare il rischio di vittimizzazione secondaria o ripetuta, di ritorsioni o di intimidazioni: QUATTROCOLO, Serena, Vulnerabilità e individual assessment: l'evoluzione dei parametri di identificazione, in BARGIS, Marta - BELLUTA, Hervè (a cura di), Vittime di reato e sistema penale. La ricerca di nuovi equilibri, Torino, 2017, p. 311 ss. V. anche CASSIBBA, Fabio, Le vittime di genere alla luce 
E così, è la legge a dover definire l'ambito in cui è riconosciuto alla vittima un diritto di informazione e di interlocuzione attraverso il recupero di una dimensione descrittiva del concetto di violenza alla persona, che non scarichi sul giudice compiti che non gli appartengono, entrando così in rotta di collisione con una serie di valori costituzionali centrali per il diritto penale, primo fra tutti proprio il principio di legalità115. Si potrebbe prospettare una definizione della categoria dei delitti commessi con violenza alla persona che contempli le conseguenze determinate dall'azione violenta (entità e tipologia del danno, prognosi su possibili ricadute negative), sulla falsariga dell'approccio adottato dalla direttiva 2012/29/UE e di quanto già affermato da parte della giurisprudenza di legittimità ${ }^{116}$.

È sempre la legge a dover costruire un modello normativo per l'intervento della vittima nella vicenda de libertate in cui venga adeguatamente considerato il diverso peso che hanno ruoli, prerogative, diritti, bisogni. Senza dubbio alcuno, vanno garantiti i diritti informativi della vittima sull'evoluzione dello status custodiae, anche al fine di una sua partecipazione al procedimento ex art. 299 c.p.p., ma vanno eliminati i doveri di notificazione imposti all'accusato affidandoli al giudice, garante della libertà del prevenuto e soprattutto il destinatario (nel sub procedimento di revoca) del contributo che l'offeso eventualmente potrà fornire per la decisione. La cornice entro cui muoversi c'è già, l'art. 299 comma 3-bis c.p.p.: prima di provvedere in ordine alla revoca o alla sostituzione delle misure coercitive il giudice non soltanto «sente il pubblico ministero», ma informa anche la vittima che può presentare memorie ai sensi dell'art. 121 c.p.p. In questo modo l'informazione funzionale all'intervento sarebbe sempre assicurata (anche nei casi di procedibilità ex officio) e malgrado si assista ad un appesantimento della procedura, questo graverebbe sull'organo giurisdizionale, non incidendo in alcun modo sui tempi e sulle garanzie che debbono

della Convenzione di Lanzarote e di Istanbul, in BARGIS, Marta - BELLUTA, Hervè (a cura di), Vittime di reato e sistema penale. La ricerca di nuovi equilibri, Torino, 2017, p. 69.

115 VIGANÒ, Francesco, La tutela penale della libertà individuale, vol. I, L'offesa mediante violenza, Milano, 2002, p. 250.

116 V. supra, § 2. 
connotare l'incidente cautelare pro libertate: un peso che il sistema nel suo complesso è in grado di reggere.

L'attenzione riservata all'offeso non deve diventare un "pericoloso arnese" in grado di scardinare il due process, l'archetipo processuale che trova nelle tutele apprestate alla libertà personale dell'imputato il suo baricentro e che rappresenta una conquista irrinunciabile per i moderni sistemi di giustizia penale. La soddisfazione dei bisogni di sicurezza e protezione della vittima non si ottiene sottraendo diritti all'imputato, ma delineando un modello rigoroso di pesi e contrappesi di cui si fa garante l'organo giurisdizionale. La latitudine del protagonismo della vittima in questo contesto non può che restare nel cono d'ombra dell'azione degli organi di giustizia, sotto l'ala protettiva del giudice, chiamato ad esercitare il suo munus entro le strette maglie di una normativa che continua a rappresentare il miglior presidio per la libertà del prevenuto.

\section{Bibliografia}

ALLEGREZZA, Silvia, La riscoperta della vittima nella giustizia penale europea, in AA.VV., Lo scudo e la spada. Esigenze di protezione e poteri delle vittime nel processo penale tra Europa e Italia, Torino, 2012, p. 1.

ALLEGREZZA, Silvia, Il ruolo della vittima nella direttiva 2012/29/UE, in AA.VV., Lo statuto europeo delle vittime di reato. Modelli di tutela tra diritto dell'Unione e buone pratiche nazionali, a cura di LUPARIA, Luca, Padova, 2015, p. 3.

AMOROSO, Maria Cristina, La nozione di delitti commessi con violenza alla persona: il primo passo delle Sezioni Unite verso un nuovo viaggio, in Cass. pen., 2016, p. 3714.

BARGIS, Marta - BELLUTA, Hervè, La direttiva 2012/29/UE: diritti minimi della vittima nel processo penale, in BARGIS, Marta - BELLUTA, Hervè (a cura di), Vittime di reato e sistema penale. La ricerca di nuovi equilibri, Torino, 2017, p. 15.

BELLUTA, Hervè, Revoca o sostituzione di misura cautelare e limiti al coinvolgimento della vittima, in Dir. pen. cont., 28 novembre 2013.

BONINI, Valentina, Il sistema di protezione della vittima e i suoi riflessi sulla libertà personale, Padova, 2018. 
BONTEMPELLI, Manfredi, Novità nelle procedure di revoca e sostituzione, in DIDDI, Alessandro - GERACI, Rosa Maria (a cura di), Misure cautelari ad personam in un triennio di riforme, Torino, 2015, p. 151.

BRESSANELLI, Caty, La "violenza di genere" fa il suo ingresso nella giurisprudenza di legittimità: le Sezioni Unite chiariscono l'ambito di applicazione dell'art. 408 co. 3-bis c.p.p., in Dir. pen. cont., 21 giugno 2016.

BUZZELLI, Silvia, La fragilità probatoria del dichiarante minorenne e la giustizia penale in Europa, in CESARI, Claudia (a cura di), Il minorenne fonte di prova nel processo penale, Milano, 2015, p. 1.

CARACENI, Lina, Misure cautelari pro victima e diritti di libertà dell'accusato: a proposito di una convivenza faticosa, in Arch. n. proc. pen., 2017, p. 254.

CASSIBBA, Fabio, Le vittime di genere alla luce della Convenzione di Lanzarote e di Istanbul, in BARGIS, Marta - BELLUTA, Hervè (a cura di), Vittime di reato e sistema penale. La ricerca di nuovi equilibri, Torino, 2017, p. 67.

CATALANO, Elena, La tutela della vittima nella direttiva 2012/29/UE e nella giurisprudenza delle Corti europee, in Riv. it. dir. proc. pen., 2014, p. 1789.

CIAMPI, Stefano, Il diritto di difesa e all'informazione, in BARGIS, Marta BELLUTA, Hervè (a cura di), Vittime di reato e sistema penale. La ricerca di nuovi equilibri, Torino, 2017, p. 241.

CORDERO, Franco, Procedura penale, Torino, 2012.

DALIA, Gaspare, I presupposti dell'obbligo di notifica alla persona offesa della richiesta di revoca o sostituzione della misura cautelare personale, in Proc. pen. giust., 2018, 2, p. 305.

DE SIMONE, Giulio, voce Violenza, in Enc. dir., 1993, vol. XLVI, p. 881.

DIDDI, Alessandro, Chiaroscuri nella nuova disciplina sulla violenza di genere, in Proc. pen. giust., 2014, p. 98.

DI NUZZO, Viviana, Giudizio cautelare e tutela della persona offesa, tra istanze di protezione e prospettive di partecipazione attiva, in Legislaz. pen., 29.7.2020.

GIALUZ, Mitja, Lo statuto europeo delle vittime vulnerabili, in AA.VV., Lo scudo e la spada. Esigenze di protezione e poteri delle vittime nel processo penale tra Europa e Italia, Torino, 2012, p. 59.

GUERRA, Mariaemanuela, Questioni in tema di diritti di informazione ed interlocuzione della persona offesa nell'incidente cautelare, in Cass. pen., 2017, p. 2536. 
IASEVOLI, Clelia, Pluralismo delle fonti e modifiche al c.p.p. per i delitti commessi con violenza alla persona, in Dir. pen. e proc., 2013, p. 1390.

LORUSSO, Sergio, Le conseguenze del reato. Verso un protagonismo della vittima nel processo penale?, in Dir. pen. proc., 2013, p. 883.

LUPARIA, Luca, Quale posizione per la vittima nel modello processuale italiano?, in AA.VV., Lo scudo e la spada. Esigenze di protezione e poteri delle vittime nel processo penale tra Europa e Italia, Torino, 2012, p. 20.

MARCHETTI, Filippo, L'intervento dell'offeso nel procedimento di revoca e sostituzione di una misura cautelare personale nuovamente sotto la lente della Suprema Corte, in Dir. pen. cont. 2019, 6, p. 121.

MORELLI, Francesco, Commento all'art. 9 d.l. 23 febbraio 2009, n. 11, in Legislaz. pen., 2009, p. 496.

ORLANDI, Renzo, I diritti della vittima in alcune particolari modalità di definizione del rito penale, in BARGIS Marta - BELLUTA Hervè (a cura di), Vittime di reato e sistema penale. La ricerca di nuovi equilibri, Torino, 2017, p. 167.

PATANÈ, Vania, Percorsi di giustizia riparativa nel sistema penale italiano, in BARGIS, Marta - BELLUTA, Hervè (a cura di), Vittime di reato e sistema penale. La ricerca di nuovi equilibri, Torino, 2017, p. 545 ss.

PAULESU, Pier Paolo, Vittima del reato e processo penale: uno sguardo d'insieme (informazioni, diritti, tutele), in BARGIS, Marta - BELLUTA, Hervè (a cura di), Vittime di reato e sistema penale. La ricerca di nuovi equilibri, Torino, 2017, p. 127.

POTETTI, Domenico, Il nuovo art. 299 c.p.p. dopo il decreto legge n. 93 del 2013, in Cass. pen., 2014, p. 975.

PROCACCINO, Angela, L'avvento della persona offesa nelle dinamiche custodiali, in DIDDI, Alessandro - GERACI, Rosa Maria (a cura di), Misure cautelari ad personam in un triennio di riforme, Torino, 2015, p. 89.

QUATTROCOLO, Serena, Vulnerabilità e individual assessment: l'evoluzione dei parametri di identificazione, in BARGIS, Marta - BELLUTA, Hervè (a cura di), Vittime di reato e sistema penale. La ricerca di nuovi equilibri, Torino, 2017, p. 297.

RAFARACI, Tommaso, Tutela della vittima nel sistema penale delle garanzie, in Criminalia, 2010, p. 260.

RECCHIONE, Sandra, La vittima cambia il volto del processo penale: le tre parti "eventuali", la testimonianza dell'offeso vulnerabile, la mutazione del principio di oralità, in Dir. pen. cont., 16 gennaio 2017. 
RIZZATO, Emma, L. 119/13, le novità in materia di contrasto al c.d. femminicidio, in Quest. giust., 28 ottobre 2013.

ROMANELLI, Bartolomeo, Omessa notifica alla persona offesa della richiesta di revoca o sostituzione di misura cautelare coercitiva: problemi definitori e rimedi in sede di impugnazione, in Cass. pen., 2017, p. 4429.

SECHI, Paola, Vittime di reato e processo penale: il contesto sovranzionale, in Cass. pen., 2017, p. 850 e 1236.

SEPE, Giuseppe, Violenza di genere e consultazione della persona offesa nelle vicende estintive delle misure cautelari, in Dir. pen. cont., 9 luglio 2014.

SPAGNOLO, Paola, Nuovi diritti informativi per la vittima di reato, in Legislaz. pen., 4-7-2016.

SPAGNOLO, Paola, Vittima di reato e diritto all'informazione: un binomio insoddisfacente, in Cass. pen., 2017, p. 3482.

VALENTINI, Elena, Il ruolo della persona offesa nella procedura di revoca e sostituzione delle misure cautelari personali, in CURI, Francesca (a cura di), Ordine pubblico e sicurezza nel governo della città, Bologna, 2016, p. 225.

VENTUROLI, Marco, La tutela della vittima nelle fonti europee, in Dir. pen. cont., 2012, f. 3-4, p. 86.

VENTUROLI, Marco, La vittima nel sistema penale: dall'oblio al protagonismo?, Napoli, 2015.

VICOLI, Daniele, La “ragionevole durata” delle indagini, Torino, 2012.

VIGANÒ, Francesco, La tutela penale della libertà individuale, vol. I, L'offesa mediante violenza, Milano, 2002.

ZACCHÈ, Francesco, Il sistema cautelare a protezione della vittima, in BARGIS, Marta - BELLUTA, Hervè (a cura di), Vittime di reato e sistema penale. La ricerca di nuovi equilibri, Torino, 2017, p. 419.

ZARCONE, Chiara, La vittima: quale spazio nel processo penale, in Quest. giust., 7 ottobre 2016. 


\section{Additional information and author's declarations (scientific integrity)}

Conflict of interest declaration: the author confirms that there are no conflicts of interest in conducting this research and writing this article.

Declaration of authorship: all and only researchers who comply the authorship requirements of this article are listed as authors; all coauthors are fully responsible for this work in its entirety.

Declaration of originality: the author assures that the text here published has not been previously published in any other resource and that future republication will only take place with the express indication of the reference of this original publication; she also attests that there is no third party plagiarism or self-plagiarism.

\section{Editorial process dates}

(http://www.ibraspp.com.br/revista/index.php/RBDPP/about/editorialPolicies)

- Submission: 02/08/2021

- Desk review and plagiarism check: 02/08/2021

- Review 1: 01/09/2021

- Review 2: 21/09/2021

- Preliminary editorial decision: 21/09/2021

- Correction round return: 01/10/2021

- Final editorial decision: 01/10/2021

\section{Editorial team}

- Editor-in-chief: 1 (VGV)

- Associated-editor: 2 (BC, LG)

- Reviewers: 2 


\section{HOW TO CITE (ABNT BRAZIL):}

CARACENI, Lina. La vittima nel procedimento de libertate: i precari equilibri di un nuovo protagonismo ancora troppo poco meditato. Revista Brasileira de Direito Processual Penal, vol. 7, n. 3, p. 17831824, set./dez. 2021. https://doi.org/10.22197/rbdpp.v7i3.632 